\title{
THE SPECTRUM OF TWO-PARTICLE BOUND STATES OF TRANSFER MATRICES OF GIBBS FIELDS. PART 3: FIELDS ON THE THREE-DIMENSIONAL LATTICE
}

\author{
E. L. LAKSHTANOV AND R. A. MINLOS
}

\begin{abstract}
In this paper, which continues our earlier papers, we investigate so-called "adjacent" bound states (that is, bound states which only appear in a neighbourhood of special values of the total quasimomentum of the system) of the transfer matrix of the general spin model on the 3-dimensional lattice in its two-particle subspace for high temperatures $T=1 / \beta$. The case of double non-degenerate extrema of the "symbol" $\omega_{\Lambda}(k), \Lambda \in \mathbb{T}^{2}, k \in \mathbb{T}^{2}$, is studied. The corresponding points $\Lambda$ are situated on certain "double" curves on the torus $\mathbb{T}^{2}$. We also study the case of degenerate extrema $\omega_{\Lambda}(k)$ situated on caustic curves on the torus. In the first case, conditions under which adjacent levels appear are indicated and the size of a neighbourhood of "double" curves where these levels "live" is estimated. In the second case, it is shown that for a degenerate extremum of $\omega_{\Lambda}(k)$ "with general position" there are no adjacent levels in a neighbourhood of caustics.
\end{abstract}

\section{BRIEF REMINDERS}

This paper is a continuation of our papers [1] and [2], where we explained in detail the problem concerning two-particle bound states of transfer matrices of Gibbs fields (with compact spin space consisting of more than 2 elements and with an arbitrary compactly supported interaction along "spatial" directions) in the high-temperature region $\beta=$ $1 / T \ll 1$.

Recall that in [1] the original problem, after several reductions, was reduced to the following problem. There is a family of Hilbert spaces $\left\{\mathcal{H}_{\Lambda}, \Lambda \in \mathbb{T}^{\nu}\right\}$ labelled by the points of the $\nu$-dimensional torus $\mathbb{T}^{\nu}$ :

$$
\left\{\mathcal{H}_{\Lambda}=\mathbb{C} \oplus \bar{L}_{2}^{\Lambda}, \Lambda \in \mathbb{T}^{\nu}\right\}
$$

where

$$
\bar{L}_{2}^{\Lambda}=\left\{f \in L_{2}\left(\mathbb{T}^{\nu}, d k\right): \int_{\mathbb{T}^{\nu}} f(k) d k=0 ; f(k)=f(\Lambda-k), k \in \mathbb{T}^{\nu}\right\} \subset L_{2}\left(\mathbb{T}^{\nu}, d k\right)
$$

2000 Mathematics Subject Classification. Primary 82C10; Secondary 47N55, 60G60, 82C20.

Key words and phrases. Adjacent bound states, Gibbs fields, transfer matrix, lattice, spectrum, Fredholm determinant, total quasimomentum.

The first author thanks Frau Cristel Schröder for hospitality during his visit to Technische Universität München, where the main part of this paper was completed. This research was partially supported by the Centre for Research on Optimization and Control in Fundação para a Ciência e a Tecnologia and by the European Community Fund FEDER/POCTI.

The second author thanks the Russian Foundation for Basic Research for financial support (grant no. 05-01-00449). 
(here $d k$ is the normed Haar measure on the torus $\mathbb{T}^{\nu}$ ). In each space $\mathcal{H}_{\Lambda}$, a self-adjoint operator $\mathcal{T}_{\Lambda}$ acts by the formulae

$$
\begin{aligned}
\left(\mathcal{T}_{\Lambda} F\right)_{0} & =\gamma(\Lambda) f_{0}+\int_{\mathbb{T}^{\nu}} b_{\Lambda}(p) f_{1}(p) d p, \\
\left(\mathcal{T}_{\Lambda} F\right)_{1}(k) & =\omega_{\Lambda}(k) f_{1}(k)+\int_{\mathbb{T}^{\nu}}\left(K_{\Lambda}(k, p)-\omega_{\Lambda}(p)\right) f_{1}(p) d p+f_{0} \bar{b}_{\Lambda}(k),
\end{aligned}
$$

where $F=\left(f_{0}, f_{1}(k)\right) \in \mathcal{H}_{\Lambda}$. Here,

$$
\begin{aligned}
\gamma(\Lambda) & =\gamma_{0}+O(\beta), & K_{\Lambda}(k, p) & =\beta^{2} K_{\Lambda}^{0}(k, p)+O\left(\beta^{3}\right), \\
b_{\Lambda}(p) & =\beta b_{\Lambda}^{0}(p)+O\left(\beta^{2}\right), & \omega_{\Lambda}(k) & =\omega_{0}+\beta \omega_{\Lambda}^{(1)}(k)+O\left(\beta^{2}\right),
\end{aligned}
$$

and $\gamma_{0}, K_{\Lambda}^{0}(k, p), b_{\Lambda}^{0}(p), \omega_{0}, \omega_{\Lambda}^{(1)}(k)$ are independent of $\beta$. The notation $O\left(\beta^{k}\right)$ means a quantity with growth less than $<C \beta^{k}$ (uniformly with respect to the other variables), where $C$ is a constant independent of $\beta$. Furthermore, $K_{\Lambda}(k, p)=\bar{K}_{\Lambda}(p, k)$ and $K_{\Lambda}(k, p)=K_{\Lambda}(\Lambda-k, p)$, and also $b_{\Lambda}(k)=b_{\Lambda}(\Lambda-k)$ and $\omega_{\Lambda}(k)=\omega_{\Lambda}(\Lambda-k)$. In addition,

$$
\omega_{0} \neq \gamma_{0} .
$$

Finally, we assume that the functions $\omega_{\Lambda}(k), \omega_{\Lambda}^{(1)}(k), b_{\Lambda}(k), b_{\Lambda}^{0}(k)$ have analytic continuations with respect to the variable $k \in \mathbb{T}^{2}$ to a sufficiently large complex neighbourhood $W_{A}^{2}$ of the torus $\mathbb{T}^{2}$; here, $W_{A}^{2}=W_{A}^{1} \times W_{A}^{1}$, where $W^{1}=\left\{z \in \mathbb{C} / \mathbb{Z}^{1} ;|\operatorname{Im} z|<A\right\}$ and $\mathbb{C} / \mathbb{Z}^{1}$ is the quotient space generated by the action of the translation group $z \rightarrow z+2 \pi m$, $m \in \mathbb{Z}^{1}$. The functions $K_{\Lambda}(k, p)$ and $K_{\Lambda}^{0}(k, p)$ also have analytic continuations to this neighbourhood with respect to the variables $k$ and $p$. We call the family of functions $\left\{\omega_{\Lambda}(k), \Lambda \in \mathbb{T}^{\nu}\right\}$ the symbol of the family $\left\{\mathcal{T}_{\Lambda}\right\}$.

It is well known (see [6]) that the spectrum of the operator $\mathcal{T}_{\Lambda}$ consists of a continuous part, which fills the segment $\left[\tau_{\Lambda}^{\min }, \tau_{\Lambda}^{\max }\right], \tau_{\Lambda}^{\text {ext }}=\operatorname{ext}_{\mathrm{k} \in \mathbb{T}^{\nu}} \omega_{\Lambda}(\mathrm{k})$, ext $=\min , \max$, and also, possibly, of eigenvalues. Our aim in this paper, as in the preceding ones, is to determine whether for sufficiently small $\beta$ there exist eigenvalues of the operator $\mathcal{T}_{\Lambda}$ situated outside the continuous spectrum (and if so, to indicate their location).

In [1, we showed the following.

1. For any dimension $\nu$ and for all values of $\Lambda$ and for sufficiently small $\beta$, the operator $\mathcal{T}_{\Lambda}$ has an eigenvalue $\varepsilon_{b}$ of the form

$$
\varepsilon_{b}=\gamma_{0}+O\left(\beta^{2}\right)
$$

(an isolated level situated at a finite distance (by (1.2) and (1.3) ) from the continuous spectrum of $\mathcal{T}_{\Lambda}$ ).

2. Outside a $D \beta^{2}$-neighbourhood of the continuous spectrum there are no other eigenvalues of the operator $\mathcal{T}_{\Lambda}$; here $D$ is some constant independent of $\beta$ and $\Lambda$.

3. For any $\delta>0$ there exists $\beta_{0}=\beta_{0}(\delta)$ such that for all $\beta<\beta_{0}$ and for $\Lambda$ lying outside the $\delta$-neighbourhood of certain special values (see below) the operator $\mathcal{T}_{\Lambda}$ has no eigenvalues apart from $\varepsilon_{\beta}$.

In [2], neighbourhoods of the special values of $\Lambda$ were studied in the case $\nu=1$ (that is, in the case of fields on the two-dimensional lattice) and it was shown that additional eigenvalues (so-called "adjacent" levels) can appear only in a neighbourhood of the socalled double values of $\Lambda$, that is, those $\Lambda$ (denoted by $\Lambda^{\text {mult }}$ ) for which the function $\omega_{\Lambda}(k)$ attains its global maximum or global minimum at two points. Furthermore, in [2], we indicated a sufficient condition for the appearance of an adjacent level, and we gave an estimate for the size of a neighbourhood where this level "lives".

In the present paper, adjacent levels of $\mathcal{T}_{\Lambda}$ are studied in the case of dimension $\nu=2$. 


\section{BASIC RESULTS ON ADJACENT LEVELS AND ASSUMPTIONS ON THE FAMILY $\left\{\omega_{\Lambda}, \Lambda \in \mathbb{T}^{\nu}\right\}$ IN THE CASE $\nu=2$}

Before stating the results on adjacent levels of the family of operators $\mathcal{T}_{\Lambda}$, we describe the location and the nature of the special values of $\Lambda$ for the symbol $\left\{\omega_{\Lambda}, \Lambda \in \mathbb{T}^{\nu}\right\}$ for $\nu=2$.

2.1. Special values of $\Lambda$. As we have already mentioned, adjacent levels of $\mathcal{T}_{\Lambda}$ for small $\beta$ can appear only if $\Lambda$ lies inside some small neighbourhood of special values of this parameter determined by the family of functions $\omega_{\Lambda}(k)$. These special values are either those $\Lambda$ at which one of the extrema of the function $\omega_{\Lambda}(\cdot)$ degenerates (we call these $\Lambda$ caustic values 1 (see [4, vol. 2]) and denote them by $\Lambda^{\text {caust }}$ ) or the $\Lambda$ for which the function $\omega_{\Lambda}(\cdot)$ has several coinciding extrema (we call these $\Lambda$ multiple values and denote them by $\left.\Lambda^{\text {mult }}\right)$. Here we introduce assumptions on the structure of sets of special values. Note that the set of special values divides naturally into two subsets. One is connected with minimal values of $\omega_{\Lambda}(k)$ (degenerate minima, multiple minima), which we denote by $\eta^{\text {min }} \subset \mathbb{T}^{2}$; the other is connected with maximal values and we denote it by $\eta^{\max }$. For definiteness, we describe the special values in $\eta^{\text {min }}$ connected with minima of $\omega_{\Lambda}(k)$ below, and corresponding to these we state results on the adjacent levels that lie below the continuous spectrum. The case of adjacent levels situated above the continuous spectrum (and the set $\eta^{\text {max }}$ of special values of $\Lambda$ connected with maximal values of the family $\left.\left\{\omega_{\Lambda}(k)\right\}\right)$ can be investigated in a similar fashion. First we describe the nature of possible critical points of the functions $\left\{\omega_{\Lambda}(k)\right\}$.

2.2. Critical points of $\omega_{\Lambda}(k)$. Since the function $\omega_{\Lambda}(k)$ is invariant under the involution of the torus $k \rightarrow \Lambda-k$ :

$$
\omega_{\Lambda}(k)=\omega_{\Lambda}(\Lambda-k)
$$

the four fixed points of this involution

$$
k^{\sigma}=\left\{\frac{\Lambda_{1}}{2}+\pi \sigma_{1}, \frac{\Lambda_{2}}{2}+\pi \sigma_{2}\right\}, \quad \sigma_{i}=0,1 ; \quad i=1,2, \quad \sigma=\left(\sigma_{1}, \sigma_{2}\right), \quad \Lambda=\left(\Lambda_{1}, \Lambda_{2}\right),
$$

where $\frac{\left|\Lambda_{i}\right|}{2} \leq \frac{\pi}{2}, i=1,2$, are critical points of the function $\omega_{\Lambda}(k)$. We call points of this form basic critical points. The other critical points of $\omega_{\Lambda}$ always occur in pairs: if $\bar{k}$ is a critical point for $\omega_{\Lambda}$, so is $\bar{k}^{\prime}=\Lambda-\bar{k}$. In what follows we regard such a pair as a single critical point and call it a pair critical point. We assume that for each $\Lambda$ the critical points of the function $\omega_{\Lambda}^{(1)}(k)$ (see the expansion (1.2)), and thus of the function $\omega_{\Lambda}$, are isolated.

2.3. Multiple and caustic values of $\Lambda$. We denote by $\Delta^{(m)} \subset \mathbb{T}^{2}$ the set of values of $\Lambda$ for which the function $\omega_{\Lambda}(k)$ has exactly $m$ coinciding minima at points $k_{1}, \ldots, k_{m}$, $k_{i} \in \mathbb{T}^{2}$ (we call these values $m$-multiple values of $\Lambda$ ).

We assume that in the case $\nu=2$ the function $\omega_{\Lambda}(k)$ has no values of multiplicity higher than three (that is, $\Delta^{(m)}=\varnothing$ for $m>3$ ). The set $\Delta^{(3)}$ consists of finitely many values $\left\{\Lambda_{\text {mult }, j}^{(3)}\right\}$, and the set $\Delta^{(2)}$ of finitely many double curves $\left\{\gamma_{\text {mult }, i}\right\}$, the ends of which either coincide with triple values (with multiplicity three) or are caustic values (see below).

We assume that the set of caustic values of $\Lambda \in \mathbb{T}^{2}$ consists of finitely many curves $\xi_{j}^{\text {caust }}$ (elementary caustics; see [3]) and in addition, possibly, finitely many isolated values

\footnotetext{
${ }^{1}$ We deviate somewhat from the generally accepted terminology: caustic values of parameters are usually defined as those values at which some critical points of the function $\omega_{\Lambda}(k)$ degenerate, rather than only its extremum points.
} 
$\Lambda_{\text {isol }}^{\text {caust }}$. For $\Lambda$ in the interior of a caustic $\xi_{j}^{\text {caust }}$, a minimum of $\omega_{\Lambda}(k)$ is attained at one of the degenerate basic critical points $k_{\min }=k_{\text {basic }}^{\sigma}, \sigma=\left(\sigma_{1}, \sigma_{2}\right), \sigma_{i}=0,1$, of the function $\omega_{\Lambda}(k)$; furthermore, the rank of degeneration is 1 and the type is $A_{3}$ (see [3]). This means that in a neighbourhood of the point $k_{\min }$ we can choose a system of coordinates $\left\{x_{1}(k), x_{2}(k)\right\}$ (satisfying $x_{1}\left(k_{\min }\right)=x_{2}\left(k_{\min }\right)=0$ ) such that the function $\omega_{\Lambda}(k)$ takes the form

$$
\omega_{\Lambda}(k)=\omega_{\Lambda}\left(k_{\min }\right)+\left(x_{1}^{2}+x_{2}^{4}\right) \beta .
$$

For the values $\Lambda=\Lambda_{\text {end }}^{\text {caust }}$, that is, lying at the endpoints of the caustic $\xi_{j}^{\text {caust }}$, the degeneration becomes stronger: in suitable coordinates, in a neighbourhood of $k_{\min }$, the function can be written as

$$
\omega_{\Lambda}(k)=\omega_{\Lambda}\left(k_{\min }\right)+\left(x_{1}^{2}+x_{2}^{6}\right) \beta .
$$

Furthermore, the value $\Lambda_{\text {end }}^{\text {caust }}$ serves simultaneously as an endpoint of some double curve $\gamma_{\text {mult }, i}$. For the interior values of $\Lambda$ on this curve, a double minimum of $\omega_{\Lambda}(k)$ is attained at some basic and pair critical points (both minima are non-degenerate). As $\Lambda$ approaches the value $\Lambda_{\text {end }}^{\text {caust }}$ along the curve $\gamma_{\text {mult }, i}$, these points come together and merge with each other at a basic (degenerate) point.

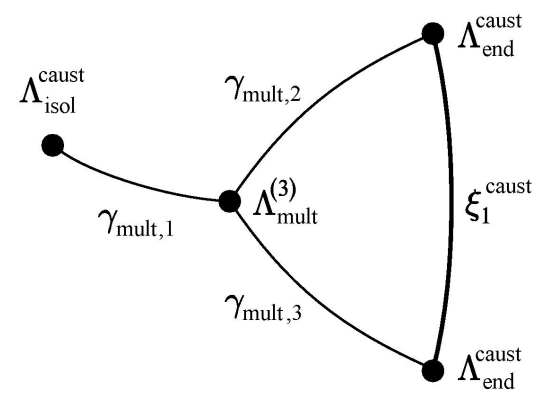

Figure 1. The simplest arrangement of special values of $\Lambda$ (the set $\eta^{\text {min }}$ or $\left.\eta^{\max }\right)$

For an isolated caustic value $\Lambda=\Lambda_{\text {isol }}^{\text {caust }}$, the function $\omega_{\Lambda}$ has a degenerate minimum attained at a pair critical point (degeneration of rank 1 and of type $A_{3}$ ); the value $\Lambda_{\text {isol }}^{\text {caust }}$ also serves as an endpoint of some double curve $\gamma_{\text {mult }, i}^{(2)}$. For values of $\Lambda$ on this curve, the global double minimum of $\omega_{\Lambda}(k)$ is attained at two different pair points. As $\Lambda$ approaches $\Lambda_{\text {isol }}^{\text {caust }}$ along the curve $\gamma_{\text {mult }, i}^{(2)}$, these points come together, merging at $\Lambda=\Lambda_{\text {isol }}^{\text {caust }}$ into a degenerate pair point (see Figure 1).

Similar assumptions are introduced with regard to the set $\eta^{\max }$ of special values of $\Lambda$ connected with maximal values of the family $\left\{\omega_{\Lambda}(k)\right\}$.

Remark 1 . The assumptions we make on the sets $\eta^{\text {ext }}$ of special values of the parameter $\Lambda$ for the family $\left\{\omega_{\Lambda}, \Lambda \in \mathbb{T}^{2}\right\}$ define a family of "general position": a "small perturbation" of this family does not change the nature of the set of special values we have described. Thus, we can assume that the set $\eta^{\text {ext }}$ with all its elements (triple points, double curves, caustic curves, and so on) is "element-wise" close to the limit set of special values for the family $\left\{\omega_{\Lambda}^{(1)}(k)\right\}$ (which no longer depends on $\beta$; see (1.2)), so that the elements of the limit set of special values of $\Lambda$ have a structure completely analogous to that of the elements of the pre-limit set. Furthermore, naturally, triple values are at a finite distance from caustic values, as well as from the other triple values. (We say that the distance between two elements of the set $\eta^{\text {ext }}$ of special values is finite if for all sufficiently 
small $\beta$ this distance exceeds some constant $c>0$ that is independent of $\beta$.) The distance between double curves that have no common endpoints is also finite.

Remark 2. In this paper we study only the adjacent levels that "live" in neighbourhoods of double and caustic curves. The case of adjacent levels of the $\beta$-neighbourhood of triple points will be considered in a consequent publication.

\section{Statement of the Results}

3.1. Basic definitions. We denote by $M_{\Lambda}\left(k_{1}, k_{2} ; z\right)$ the kernel

$$
M_{\Lambda}\left(k_{1}, k_{2} ; z\right)=K_{\Lambda}\left(k_{1}, k_{2}\right)-\frac{b_{\Lambda}\left(k_{1}\right) \bar{b}_{\Lambda}\left(k_{2}\right)}{\gamma(\Lambda)-z}
$$

and let

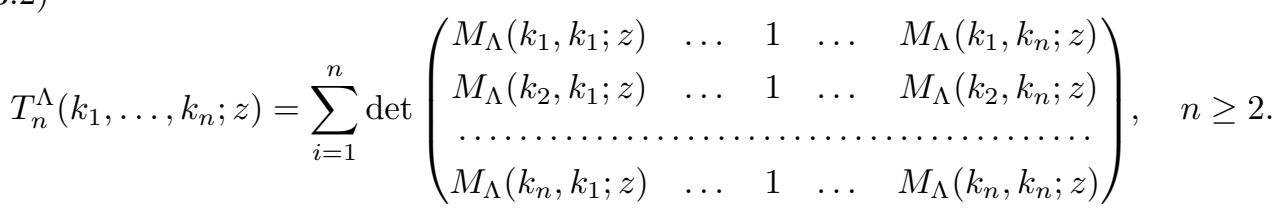

In the sum (3.2), each column of the matrix $\left\{M_{\Lambda}\left(k_{i}, k_{j} ; z\right), i, j=1, \ldots, \nu\right\}$ is consecutively replaced by a column consisting of 1 s. Since

$$
T_{n}^{\Lambda}\left(k_{1}, \ldots, k_{n} ; z\right)=\operatorname{det}\left(M_{\Lambda}+\Pi\right)-\operatorname{det} M_{\Lambda},
$$

where $M_{\Lambda}=\left\{M_{\Lambda}\left(k_{i}, k_{j} ; z\right)\right\}$ is a Hermitian matrix and the matrix $\Pi$ consists entirely of $1 \mathrm{~s}$, the function $T_{n}^{\Lambda}\left(k_{1}, \ldots, k_{n} ; z\right)$ is real (for real $z$ ). As follows from the representations (1.2), the function $T_{n}^{\Lambda}\left(k_{1}, \ldots, k_{n} ; z\right)$ has the form

$$
T_{n}^{\Lambda}\left(k_{1}, \ldots, k_{n} ; z\right)=\left(\beta^{2}\right)^{n-1} T_{n, 0}^{\Lambda}\left(k_{1}, \ldots, k_{n} ; z\right)(1+O(\beta)),
$$

where $T_{n, 0}^{\Lambda}\left(k_{1}, \ldots, k_{n} ; z\right)$ is defined by formula (3.2) in which the function $M\left(k_{1}, k_{2} ; z\right)$ is replaced by the following function (which is independent of $\beta$; see (1.2)):

$$
M_{\Lambda}^{0}\left(k_{1}, k_{2}\right)=K_{\Lambda}^{0}\left(k_{1}, k_{2}\right)-\frac{b_{\Lambda}^{0}\left(k_{1}\right) \bar{b}_{\Lambda}^{0}\left(k_{2}\right)}{\gamma_{0}-z} .
$$

Next, for each double value $\Lambda_{\text {mult }}^{(2)}=\Lambda \in \Delta^{(2)}$ we define the quantity

$$
B_{\Lambda}=\frac{1}{\beta^{2}} T_{2}^{\Lambda}\left(\bar{k}_{1}, \bar{k}_{2}, \tau_{\Lambda}^{\mathrm{ext}}\right)( \pm 1)
$$

where $\bar{k}_{\sigma}=k_{\sigma}^{\text {ext }}(\Lambda), \sigma=1,2$, are the points at which a double extremum of $\omega_{\Lambda}$ is attained, the + sign is chosen when this extremum is a minimum ext $=$ min, and the - sign is chosen when ext $=\max$ (if one or both extrema are attained at pair points, then $\bar{k}_{\sigma}$ is one of these points).

It follows from the representation (3.4) and (3.3) that for small $\beta$ we have

$$
B_{\Lambda}=B_{\Lambda, 0}+O(\beta), \quad \Lambda \in \Delta^{(2)},
$$

where $B_{\Lambda, 0}=T_{2,0}^{\Lambda}\left(\bar{k}_{1}, \bar{k}_{2}, \tau_{\Lambda}^{\text {ext }}\right)( \pm 1)$ is independent of $\beta$.

We call a value $\Lambda_{\text {mult }}^{(2)}=\Lambda \in \Delta^{(2)}$ a threshold value if $B_{\Lambda, 0}<0$ (and, consequently, $B_{\Lambda}<0$ for small $\beta$ ). The set of threshold values is divided into finitely many intervals situated on double curves $\gamma_{\text {mult }, i}^{(2)} \subset \Delta^{(2)}$. The complementary intervals on the $\gamma_{\text {mult }, i}^{(2)}$, where $B_{\Lambda, 0}>0$, are called non-threshold intervals. The values $\Lambda_{\text {mult }}^{(2)} \in \gamma_{\text {mult }}^{(2)}$ where $B_{\Lambda, 0}=0$ are called boundary values. 
In what follows, after excluding finite neighbourhoods of triple points, we describe some domains of values of $\Lambda$ on the torus $\mathbb{T}^{2}$ in which adjacent levels certainly "live", and those domains in which there are no adjacent levels. However, it turns out that for some other (very small) domains of the torus we cannot say whether or not they have adjacent levels: in particular, these domains include neighbourhoods of endpoint values or isolated caustic values adjoining a threshold interval, as well as neighbourhoods of boundary values $\Lambda_{\text {mult }}^{(2)} \in \gamma_{\text {mult }}^{(2)}$ on double curves (where $B_{\Lambda, 0}=0$ ). We denote the set of all these exceptional $\Lambda \in \eta^{\min }$ by $\widehat{\eta}_{\mathrm{exc}}^{\min } \subset \eta^{\min }$.

Thus, we choose an arbitrary $\delta>0$ and cover each exceptional value $\Lambda \in \widehat{\eta}_{\text {exc }}^{\min }$ by a neighbourhood $V_{\delta}(\Lambda)$ of radius $\delta$. Let

$$
V_{\delta}\left(\widehat{\eta}_{\mathrm{exc}}^{\min }\right)=\bigcup_{\Lambda \in \widehat{\eta}_{\mathrm{exc}}^{\min }} V_{\delta}(\Lambda)
$$

be the $\delta$-neighbourhood of the whole set $\widehat{\eta}_{\text {exc }}^{\min }$ of exceptional values. Let

$$
V_{\delta}\left(\Delta_{\text {mult }}^{(3)}\right)=\bigcup_{\Lambda \in \Delta_{\text {mult }}^{(3)}} V_{\delta}(\Lambda)
$$

be the $\delta$-neighbourhood of the set of triple values of $\Lambda$. We denote by $\eta_{\delta}^{\min } \subset \eta^{\text {min }}$ the set of special values that are not contained in the neighbourhoods $V_{\delta}\left(\widehat{\eta}_{\text {exc }}^{\min }\right)$ and $V_{\delta}\left(\Delta_{\text {mult }}^{(3)}\right)$ :

$$
\eta_{\delta}^{\min }=\eta^{\min } \backslash\left(\eta^{\min } \cap\left(V_{\delta}\left(\eta_{\mathrm{exc}}^{\min }\right) \cup V_{\delta}\left(\Delta_{\text {mult }}^{(3)}\right)\right)\right) .
$$

Clearly, the set $\eta_{\delta}^{\text {min }}$ consists of the union of intervals $\rho_{\text {mult }}^{(2)} \subset \gamma_{\text {mult }}^{(2)}$ that lie on double curves and intervals $\pi^{\text {caust }}$ that lie on caustics. Each of the intervals $\rho_{\text {mult }}^{(2)}$ is a part of some threshold or non-threshold interval and correspondingly we call them truncated double (threshold or non-threshold) intervals. Truncated caustic intervals $\pi^{\text {caust }}$ are defined in a similar fashion. Let $0<\delta_{1}$ be a certain number that we choose below so that $\delta_{1} \beta^{2}<\frac{1}{3} \delta$. We consider the $\beta^{2} \delta_{1}$-neighbourhood $V_{\beta^{2} \delta_{1}}\left(\rho_{\text {mult }}^{(2)}\right)$ of a truncated double interval, as well as the $\beta^{2} \delta_{1}$-neighbourhood $V_{\beta^{2} \delta_{1}}\left(\pi^{\text {caust }}\right)$ of a truncated caustic interval.

Thus, we shall describe the appearance (or absence) of adjacent levels of the operator $\mathcal{T}_{\Lambda}$ for values of $\Lambda$ contained in the following sets:

1. the $\beta^{2} \delta_{1}$-neighbourhoods of truncated double intervals $\rho_{\text {mult }}^{(2)}$;

2 . the $\beta^{2} \delta_{1}$-neighbourhoods of truncated caustic intervals $\pi^{\text {caust }}$.

We consider each of these cases separately.

3.2. Truncated intervals on double curves. Let $\rho \subset \gamma_{\text {mult }}^{(2)}$ be some truncated interval, and $V_{\delta_{1} \beta^{2}}$ its $\delta_{1} \beta^{2}$-neighbourhood. We assume that $\beta$ is chosen to be so small that the following conditions hold.

1. For any $\Lambda \in V_{\delta_{1} \beta^{2}}$ the function $\omega_{\Lambda}(k)$ has two non-degenerate minima attained at points $k_{1}^{\min }(\Lambda)$ and $k_{2}^{\min }(\Lambda)$, one of which is global, and the other, generally speaking, is local, and both of them are close to the coincident global minima on the double curve. We denote these minima by

$$
\tau_{\Lambda}^{\sigma}=\omega_{\Lambda}\left(k_{\sigma}^{\min }(\Lambda)\right), \quad \sigma=1,2 ; \quad \tau_{\Lambda}^{\min }=\min \left\{\tau_{\Lambda}^{(1)}, \tau_{\Lambda}^{(2)}\right\} .
$$

We assume that

$$
\beta \kappa_{2} d(\Lambda, \rho)>\left|\tau_{\Lambda}^{(1)}-\tau_{\Lambda}^{(2)}\right|>\beta \kappa_{1} d(\Lambda, \rho)
$$

for all $\Lambda \in V_{\delta_{1} \beta^{2}}(\rho)$, where $\kappa_{2}>\kappa_{1}>0$ are two constants independent of $\beta$ and $\Lambda$, while $d(\Lambda, \rho)$ is the distance from $\Lambda$ to the interval $\rho$. Thus, for $\Lambda \in V_{\delta_{1} \beta^{2}}$ 


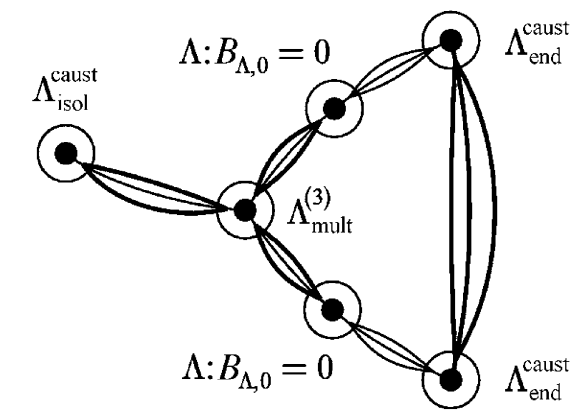

$$
\begin{aligned}
& \Longleftrightarrow-\text { truncated threshold interval with its } \delta_{1} \beta^{2} \text {-neighbourhood } \\
& - \text { truncated non-threshold interval with its } \delta_{1} \beta^{2} \text {-neighbourhood } \\
& -\delta_{1} \beta^{2} \text {-neighbourhood of a truncated caustic interval } \\
& -\delta \text {-neighbourhoods of the points } \Lambda_{\text {isol }}^{\text {caust }}, \Lambda_{\text {end }}^{\text {caust }}, \Lambda_{\text {mult }}^{(3)} \\
& \text { and of boundary } \Lambda \text { in } \Lambda_{\text {mult }}^{(2)} \text {, where } B_{\Lambda, 0}=0 .
\end{aligned}
$$

FiguRE 2. The arrangement of truncated intervals on double and caustic curves

we have

$$
\left|\tau_{\Lambda}^{(1)}-\tau_{\Lambda}^{(2)}\right| \leq \beta^{3} \kappa_{2} \delta_{1} .
$$

2. For the points $k_{\sigma}^{\min }(\Lambda), \Lambda \in V_{\delta_{1} \beta^{2}}(\rho)$, we can choose their $\varepsilon$-neighbourhoods $U_{\varepsilon}^{\sigma}$ of the form

$$
U_{\varepsilon}^{\sigma}=\left\{k: \omega_{\Lambda}(k)-\tau_{\Lambda}^{\sigma}<\varepsilon \beta\right\}
$$

so small that the Hessian of the function $\omega_{\Lambda}(k)$ does not change its sign in each of these neighbourhoods, they do not contain other critical points of this function, and in $U_{\varepsilon}^{\sigma}$ there is a system of coordinates $x^{\sigma}(k)=\left(x_{1}^{\sigma}(k), x_{2}^{\sigma}(k)\right)$ in which the function $\omega_{\Lambda}(k)$ is represented in the form

$$
\omega_{\Lambda}(k)=\omega_{\Lambda}\left(k_{\sigma}^{\min }(\Lambda)\right)+\frac{\beta}{2}\left(\left(x_{1}^{\sigma}\right)^{2}+\left(x_{2}^{\sigma}\right)^{2}\right),
$$

while in the new coordinates $\left(x_{1}^{\sigma}, x_{2}^{\sigma}\right)$, the neighbourhood $U_{\varepsilon}^{\sigma}$ becomes the disc

$$
\widetilde{U}_{\varepsilon}=\left\{\left(x_{1}^{\sigma}\right)^{2}+\left(x_{2}^{\sigma}\right)^{2}<2 \varepsilon\right\} .
$$

Furthermore, the map

$$
f_{\sigma}^{\Lambda}: \widetilde{U}_{\varepsilon}^{\sigma} \rightarrow U_{\varepsilon}^{\sigma}: k=f_{\sigma}(x), \quad x \in \widetilde{U}_{\varepsilon}^{\sigma}
$$

is infinitely smooth and non-degenerate, while its Jacobian $J_{\Lambda}^{\sigma}(x)$ satisfies the estimate

$$
0<C_{1}<J_{\Lambda}^{\sigma}(x)<C_{2},
$$

where $C_{1}, C_{2}$ are constants independent of $\Lambda$ and $\beta$. Outside the neighbourhoods $U_{\varepsilon}^{\sigma}$, the function $\omega_{\Lambda}(k)$ satisfies the inequality

$$
\max _{\sigma}\left\{\omega_{\Lambda}(k)-\tau_{\Lambda}^{\sigma}\right\}>\varepsilon \beta, \quad k \in \mathbb{T}^{2} \backslash\left(U_{\Lambda}^{(1)} \cup U_{\Lambda}^{(2)}\right) .
$$


3. For all $\Lambda \in V_{\delta_{1} \beta^{2}}(\rho)$, the quantity

$$
B_{\Lambda}=T_{2}^{\Lambda}\left(k_{1}^{\min }(\Lambda), k_{2}^{\min }(\Lambda) ; \tau_{\Lambda}^{\min }\right) \frac{1}{\beta^{2}}
$$

can be represented in the form

$$
B_{\Lambda}=B_{\Lambda, 0}(1+O(\beta)), \quad\left|B_{\Lambda, 0}\right|>\kappa_{3}
$$

(see the similar representation for the case $\Lambda=\Lambda_{\text {mult }}^{(2)} \in \gamma_{\text {mult }}^{(2)}$ ), where $B_{\Lambda, 0}$ has fixed sign for all $\Lambda \in V_{\delta_{1} \beta}(\rho)$ and is independent of $\beta$, while $\kappa_{3}>0$ is some constant independent of $\beta$ and $\Lambda \in V_{\delta_{1} \beta^{2}}$.

Remark. Since the sign of $B_{\Lambda, 0}$ coincides with the sign of this quantity for $\Lambda \in \rho$, we can speak about a threshold $\left(B_{\Lambda, 0}<0\right)$ or non-threshold $\left(B_{\Lambda, 0}>0\right)$ neighbourhood $V_{\delta_{1} \beta^{2}}(\rho)$ of the interval $\rho$.

In what follows we consider $z$ in the domain

$$
\mathcal{F}_{D}^{\Lambda}=\left\{z \in \mathbb{C}^{1}: \operatorname{Re}\left(\tau_{\Lambda}^{\min }-z\right)>0,\left|z-\tau_{\Lambda}^{\min }\right|<D \beta^{2}\right\}
$$

on the complex $z$-plane, where $D$ is the constant mentioned in the first section 2 It is obvious that for $\Lambda \in V_{\delta_{1} \beta^{2}}(\rho)$, where $\rho$ is a truncated double interval and $z \in \mathcal{F}_{D}^{\Lambda}$, we have

$$
\left|T_{2}^{\Lambda}\left(k_{1}^{\min }(\Lambda), k_{2}^{\min }(\Lambda) ; z\right) \frac{1}{\beta^{2}}-B_{\Lambda}\right|<\kappa_{4} \beta^{2},
$$

where $\kappa_{4}>0$ is a constant independent of $\beta, \Lambda$, and $z$. In particular, it follows from (3.13) and the last inequality that

$$
\left|T_{2}^{\Lambda}\left(k_{1}^{\min }(\Lambda), k_{2}^{\min }(\Lambda) ; z\right)\right|<\frac{\kappa_{3}}{2} \beta^{2}, \quad z \in \mathcal{F}_{D}^{\Lambda},
$$

and the sign of

$$
\operatorname{Re} T_{2}^{\Lambda}\left(k_{1}^{\min }(\Lambda), k_{2}^{\min }(\Lambda) ; z\right), \quad z \in \mathcal{F}_{D}^{\Lambda},
$$

coincides with the sign of $B_{\Lambda}$. Finally, we assume that $\beta$ is chosen to be so small that for $\Lambda$ lying outside the $\delta$-neighbourhood of all the special values, the operator $\mathcal{T}_{\Lambda}$ has no adjacent levels below the continuous spectrum (as follows from the results of [1]).

\section{Theorem 3.1.}

1. Let $\rho \in \gamma_{\text {mult }}^{2}$ be some double truncated threshold interval. Then there exist two numbers $0<\mu_{1}<\mu_{2}<\delta_{1} \beta^{2}, \mu_{\sigma}=\mu_{\sigma}(\beta), \sigma=1,2$, such that for any $\Lambda \in V_{\mu_{1}}(\rho)$ in the $\mu_{1}$-neighbourhood of the interval $\rho$, the operator $\mathcal{T}_{\Lambda}$ has exactly one adjacent level $\varepsilon(\Lambda)$ such that

$$
e^{-R_{1} / \beta}>\tau_{\Lambda}^{\min }-\varepsilon(\Lambda)>0,
$$

where $R_{1}$ is a constant independent of $\Lambda$ and $\beta$. For $\Lambda \in V_{\delta_{1} \beta^{2}}(\rho) \backslash V_{\mu_{2}}(\rho)$ lying outside the $\mu_{2}$-neighbourhood of the interval $\rho$ (but inside the $\beta^{2} \delta_{1}$-neighbourhood of this interval), the operator $\mathcal{T}_{\Lambda}$ has no adjacent levels below the continuous spectrum. The numbers $\mu_{1}$ and $\mu_{2}$ can be chosen to be equal to

$$
\mu_{\sigma}=e^{-c_{\sigma} / \beta}, \quad \sigma=1,2,
$$

where $c_{1}>c_{2}>0$ are two constants independent of $\Lambda$ and $\beta$.

2. In the case where $\rho \subset \gamma_{\text {mult }}^{(2)}$ is a non-threshold truncated interval on a double curve, for all $\Lambda$ in the $\beta^{2} \delta_{1}$-neighbourhood of $\rho$, the operator $\mathcal{T}_{\Lambda}$ has no adjacent levels below the continuous spectrum.

\footnotetext{
${ }^{2}$ Recall that outside the $D \beta^{2}$-neighbourhood of its continuous spectrum, $\mathcal{T}_{\Lambda}$ has no other eigenvalues, apart from an isolated level.
} 
3.3. Case of truncated caustic intervals. Let $\pi^{\text {caust }} \subset \eta^{\text {min }}$ be a truncated caustic interval, and $V_{\delta_{1} \beta^{2}}\left(\pi^{\text {caust }}\right)$ its $\delta_{1} \beta^{2}$-neighbourhood. We assume that $\beta$ is such that the following conditions hold.

1. For $\Lambda \in V_{\delta_{1} \beta^{2}}\left(\pi^{\text {caust }}\right)$, the function $\omega_{\Lambda}(k)$ has only one minimum $\tau_{\Lambda}^{\min }$. For $\Lambda \in V_{\delta_{1} \beta^{2}}$ lying on one side of the caustic interval $\pi^{\text {caust }}$, as well as on the interval itself, this minimum is attained at some of the basic points $k^{\min }(\Lambda)=$ $k_{\Lambda}^{\text {basic }}$ (these are the same for all $\Lambda$ in this part of $V_{\delta_{1} \beta^{2}}$, which we denote by $\left.V_{\delta_{1} \beta^{2}}(+)\right)$. For $\Lambda$ lying on the other side of $\pi^{\text {caust }}$, the minimum point is a pair point $k^{\min }(\Lambda)=\left(k_{1}, k_{2}\right)$; we denote this part of the neighbourhood $V_{\delta_{1} \beta^{2}}$ by $V_{\delta_{1} \beta^{2}}(-)$. As we mentioned above, on the caustic interval $\pi^{\text {caust }}$ itself, the minimum is degenerate with degeneration type $A_{3}$.
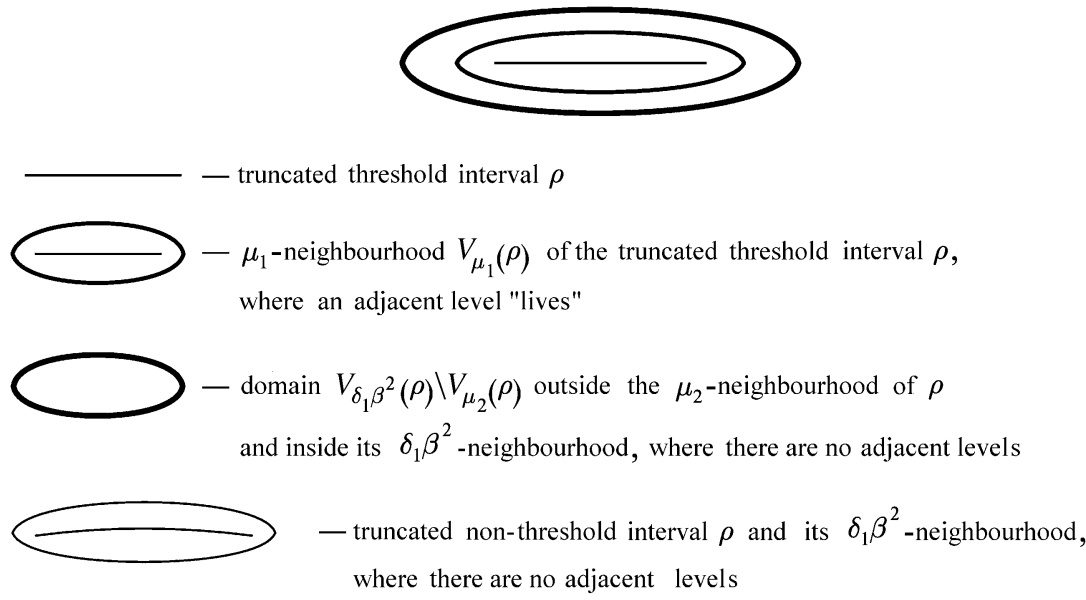

FiguRE 3. Arrangement of neighbourhoods of truncated threshold and non-threshold intervals

2. There exists a neighbourhood $U_{\varepsilon}$ of the basic point $k_{\Lambda}^{\text {basic }}$ of the form (3.8),

$$
U_{\varepsilon}=\left\{k \in \mathbb{T}^{2}:\left|\omega_{\Lambda}(k)-\omega_{\Lambda}\left(k^{\min }(\Lambda)\right)\right|<\varepsilon \beta\right\},
$$

which does not contain other critical points of $\omega_{\Lambda}(k)$, apart from, possibly, a pair point $k^{\min }(\Lambda)$. Outside this neighbourhood, the function $\omega_{\Lambda}(k)$ satisfies the condition

$$
\omega_{\Lambda}(k)-\omega_{\Lambda}\left(k_{\Lambda}^{\text {basic }}\right) \geq \varepsilon \beta, \quad k \notin U_{\varepsilon} .
$$

In the neighbourhood $U_{\varepsilon}$ we can choose a system of coordinates $x=\left(x_{1}, x_{2}\right)$, $x_{\sigma}=x_{\sigma}(k), \sigma=1,2$, with $x\left(k_{\Lambda}^{\text {basic }}\right)=0$ (generally speaking, depending on $\beta$ and $\Lambda)$ so that in these coordinates the function $\omega_{\Lambda}(k)$ is written in the form

$$
\omega_{\Lambda}(k)=\omega_{\Lambda}\left(k^{\min }(\Lambda)\right)+\beta\left(x_{1}^{2}+\xi(\Lambda) x_{2}^{2}+x_{2}^{4}\right),
$$

where $\xi(\Lambda)$ is some smooth real function of $\Lambda \in V_{\delta_{1} \beta^{2}}\left(\pi^{\text {caust }}\right)$ that vanishes for $\Lambda \in \pi^{\text {caust }}$ and takes positive values for $\Lambda \in V_{\delta_{1} \beta^{2}}(+)$, and negative values for $\Lambda \in V_{\delta_{1} \beta^{2}}(-)$; furthermore, the gradient of the function $\xi(\Lambda)$ satisfies the condition

$$
0<\kappa_{6}<\left|\nabla_{\Lambda} \xi(\Lambda)\right|<\kappa_{7}, \quad \Lambda \in V_{\delta_{1} \beta^{2}}\left(\pi^{\text {caust }}\right),
$$

where $\kappa_{6}, \kappa_{7}$ are some constants independent of $\Lambda$ and $\beta$. 
The neighbourhood $U_{\varepsilon}=U_{\varepsilon}(\Lambda)$ in the new coordinates $\left(x_{1}, x_{2}\right)=x$ is represented in the form

$$
\widetilde{U}_{\varepsilon}=\left\{\left(x_{1}, x_{2}\right): x_{1}^{2}+\xi(\Lambda) x_{2}^{2}+x_{4}^{2}<\varepsilon\right\},
$$

and the map $f: \widetilde{U}_{\varepsilon} \rightarrow U_{\varepsilon}$ taking $\left(x_{1}, x_{2}\right)$ to $k=\left(k^{(1)}, k^{(2)}\right)$ is non-degenerate and is defined by infinitely smooth functions; its Jacobian $J_{\Lambda}(x)$ satisfies estimates similar to (3.10). The maximal dilation of the map $f$,

$$
\sup _{|x|<\sqrt{\varepsilon}} \frac{\left|f(x)-k_{\Lambda}^{\text {basic }}\right|}{|x|}
$$

is bounded by a constant $F$ independent of $\Lambda$ and $\beta$. We also assume that the pair critical points $k^{\min }(\Lambda)=\left(k_{1}, k_{2}\right)$ for $\Lambda \in V_{\delta_{1} \beta^{2}}(-)$ lie inside the neighbour$\operatorname{hood} U_{\varepsilon}$.

Theorem 3.2. Let $\pi^{\text {caust }}$ be a truncated caustic interval, and let $V_{\delta_{1} \beta^{2}}\left(\pi^{\text {caust }}\right)$ be its $\delta_{1} \beta^{2}$-neighbourhood. Then for any $\Lambda \in V_{\delta_{1} \beta^{2}}\left(\pi^{\text {caust }}\right)$, the operator $\mathcal{T}_{\Lambda}$ has no adjacent levels below the continuous spectrum.

\section{Proof of Theorem 3.1}

4.1. The principal part of the Fredholm determinant. All the subsequent proofs are based on studying the asymptotic behaviour of the modified Fredholm determinant $\Delta_{\Lambda}(z)$ for $z$ close to a minimum of the function $\omega_{\Lambda}(k)$ and for sufficiently small $\beta$. As is explained, for example, in [7], it is the zeros of this determinant that give the eigenvalues of the operator $\mathcal{T}_{\Lambda}$. Recall (see [6]) that the function $\Delta_{\Lambda}(z)$ can be represented in the form of the series

$$
\Delta_{\Lambda}(z)=\int_{\mathbb{T}^{2}} \frac{d k}{\omega_{\Lambda}(k)-z}+\sum_{n=2}^{\infty} \frac{1}{n !} \int_{\mathbb{T}^{2}} \cdots \int_{\mathbb{T}^{2}} \frac{T_{n}\left(k_{1}, \ldots, k_{n} ; z\right) d k_{1} \cdots d k_{n}}{\left(\omega_{\Lambda}\left(k_{1}\right)-z\right) \cdots\left(\omega_{\Lambda}\left(k_{n}\right)-z\right)},
$$

where the functions $T_{n}\left(k_{1}, \ldots, k_{n} ; z\right)$ were introduced in (3.2). Here we establish this asymptotic behaviour in the case $\Lambda \in V_{\delta_{1} \beta^{2}}(\rho)$, where $\rho \subset \gamma_{\text {mult }}^{(2)}$ is a truncated double interval.

4.2. The first term of the series (4.1). We introduce the functions

$$
\varphi_{\Lambda}^{(\sigma)}(z)=\int_{U_{\varepsilon}^{\sigma}} \frac{d k}{\omega_{\Lambda}(k)-z}, \quad \sigma=1,2
$$

where the $U_{\varepsilon}^{\sigma}$ are the neighbourhoods of the points $k_{\sigma}^{\min }(\Lambda)$ introduced in (3.8).

Lemma 4.1. Let $\rho \subseteq \gamma_{\text {mult }}^{(2)}$ be a truncated interval on a double curve and let $\Lambda \in V_{\delta_{1} \beta^{2}}(\rho)$ and $z \in \mathcal{F}_{D}^{\Lambda}$. Then we have the representation

$$
\int_{\mathbb{T}^{2}} \frac{d k}{\omega_{\Lambda}(k)-z}=\varphi_{\Lambda}^{(1)}(z)+\varphi_{\Lambda}^{(2)}(z)+\frac{\bar{K}_{0}(\Lambda)}{\beta}+\Psi_{\Lambda}^{(0)}(z),
$$

where $\bar{K}_{0}(\Lambda)$ is a constant independent of $\beta$ and $z$, and $\Psi_{\Lambda}^{(0)}(z)$ is a bounded analytic function in $\mathcal{F}_{D}^{\Lambda}$ :

$$
\left|\Psi_{\Lambda}^{(0)}(z)\right|<C_{1},
$$

where $C_{1}>0$ is independent of $\Lambda, z$, and $\beta$.

Furthermore,

$$
\varphi_{\Lambda}^{(\sigma)}(z)=-A_{\Lambda}^{(\sigma)} \ln \left(\frac{\tau_{\Lambda}^{\sigma}-z}{\beta}\right)+\frac{K_{\sigma}(\Lambda)}{\beta}+\Psi_{\Lambda}^{(\sigma)}(z)+\Phi_{\Lambda}^{(\sigma)}(z), \quad \sigma=1,2,
$$


where

$$
A_{\Lambda}^{(\sigma)}=2 \pi\left(\operatorname{det}\left\{\left(\omega_{\Lambda}\right)_{i j}^{\prime \prime}\left(k_{\sigma}^{\min }(\Lambda)\right)\right\}\right)^{-1 / 2}
$$

$K_{\sigma}(\Lambda)$ is a constant independent of $\beta$ and $z, \Psi_{\Lambda}^{(\sigma)}(z)$ is a bounded analytic function in $\mathcal{F}_{D}^{\Lambda}$ satisfying the estimate (4.3), $\Phi_{\Lambda}^{(\sigma)}(z)$ is an analytic function in $\mathcal{F}_{D}^{\Lambda}$ admitting the estimate

$$
\left|\Phi_{\Lambda}^{(\sigma)}(z)\right|<C_{2}\left|\frac{\tau_{\Lambda}^{\sigma}-z}{\beta^{2}} \ln \left(\frac{\tau_{\Lambda}^{\sigma}-z}{\beta}\right)\right|,
$$

where $C_{2} \geq 0$ is a constant independent of $\Lambda, \beta, z$, and $\left(\omega_{\Lambda}^{\prime \prime}\right)_{i j}, i, j=1,2$, is the matrix of the second derivatives of the function $\omega_{\Lambda}(k)$.

Remark. It follows from the general construction in the neighbourhood $U_{\varepsilon}^{\sigma}$ of the coordinates $\left(x_{1}, x_{2}\right)$ in which the function $\omega_{\Lambda}(k)$ is represented in the form (3.9) (see [8]), for a "small perturbation" of this function (and its derivatives), the map $f_{\sigma}^{\Lambda}$ (see (3.10)) and its derivatives also only change a little. Hence the Jacobian $J_{\Lambda}^{\sigma}$ of this map has the form

$$
J_{\Lambda}^{\sigma}(x)=J_{\Lambda, 0}^{\sigma}(x)(1+O(\beta)),
$$

where $J_{\Lambda, 0}^{\sigma}$ is the Jacobian of the map $f_{\sigma}^{\Lambda, 0}$ constructed from the function $\omega_{\Lambda}^{(1)}(k)$ (see (1.1)). Hence the constants $A_{\Lambda}^{(\sigma)}$ have the form

$$
A_{\Lambda}^{(\sigma)}=\frac{a_{\sigma, 0}^{\Lambda}}{\beta}(1+O(\beta)),
$$

where

$$
a_{\sigma, 0}^{\Lambda}=2 \pi\left(\operatorname{det}\left\{\left(\omega_{\Lambda}^{(1)^{\prime \prime}}\right)_{i j}\left(\bar{k}_{\sigma}^{\min }(\Lambda)\right)\right\}\right)^{-1 / 2}
$$

is a constant independent of $\beta$, while $\bar{k}_{\sigma}^{\mathrm{min}}(\Lambda)$ is a minimum point of $\omega_{\Lambda}^{(1)}(k)$ close to $k_{\sigma}^{\min }(\Lambda)$.

Proof of Lemma 4.1. We represent the integral (4.2) in the form

$$
\int_{\mathbb{T}^{2}} \frac{d k}{\omega_{\Lambda}(k)-z}=\varphi_{\Lambda}^{(1)}(z)+\varphi_{\Lambda}^{(2)}(z)+\int_{\mathbb{T}^{2} \backslash\left(U_{\varepsilon}^{(1)} \cup U_{\varepsilon}^{(2)}\right)} \frac{d k}{\omega_{\Lambda}(k)-z} .
$$

First we analyse the third summand in (4.7). We observe that

$$
\frac{1}{\omega_{\Lambda}(k)-z}=\frac{1}{\omega_{\Lambda}-\tau_{\Lambda}^{\min }}-\frac{\tau_{\Lambda}^{\min }-z}{\left(\omega_{\Lambda}(k)-\tau_{\Lambda}^{\min }\right)\left(\omega_{\Lambda}(k)-z\right)} .
$$

Since

$$
\left|\omega_{\Lambda}-z\right|>\left|\omega_{\Lambda}-\tau_{\Lambda}^{\min }\right|>\varepsilon \beta
$$

outside the neighbourhoods $U_{\varepsilon}^{(1)}$ and $U_{\varepsilon}^{(2)}$ for $z \in \mathcal{F}_{D}^{\Lambda}$, while $\left|\tau_{\Lambda}^{\min }-z\right|<D \beta^{2}$ in the domain $\mathcal{F}_{D}^{\Lambda}$, the second term on the right-hand side of equality (4.8) does not exceed a constant independent of $\beta$ and $\Lambda$. Next,

$$
\omega_{\Lambda}-\tau_{\Lambda}^{\min }=\beta\left(\omega_{\Lambda}^{(1)}-\widetilde{\tau}_{\Lambda}^{\min }\right)+O\left(\beta^{2}\right),
$$

where $\widetilde{\tau}_{\Lambda}^{\min }$ is the minimal value of the function $\omega_{\Lambda}^{(1)}$. Hence, since for sufficiently small $\beta$,

$$
\omega_{\Lambda}^{(1)}-\widetilde{\tau}_{\Lambda}^{\min }>\frac{1}{2} \varepsilon
$$

outside the neighbourhoods $U_{\varepsilon}^{(1)}$ and $U_{\varepsilon}^{(2)}$, we obtain

$$
\frac{1}{\omega_{\Lambda}-\tau_{\Lambda}^{\min }}=\frac{1}{\beta\left(\omega_{\Lambda}^{(1)}-\widetilde{\tau}_{\Lambda}^{\min }\right)}(1+O(\beta))=\frac{1}{\beta\left(\omega_{\Lambda}^{(1)}-\widetilde{\tau}_{\Lambda}^{\min }\right)}+O(1) .
$$


Finally, from this we deduce that

$$
\int_{\mathbb{T}^{2} \backslash\left(U_{\varepsilon}^{(1)} \cup U_{\varepsilon}^{(2)}\right)} \frac{d k}{\omega_{\Lambda}(k)-z}=\frac{\bar{K}_{0}(\Lambda)}{\beta}+\Psi_{\Lambda}^{(0)}(z),
$$

where the function $\Psi_{\Lambda}^{(0)}(z)$ is analytic in $\mathcal{F}_{D}^{\Lambda}$ and bounded, $\left|\Psi_{\Lambda}^{(0)}(z)\right|<\widetilde{C}_{1}$ (here $\widetilde{C}_{1}>0$ is a constant independent of $\beta, \Lambda$, and $z$ ), while

$$
\bar{K}_{0}(\Lambda)=\int_{\mathbb{T}^{2} \backslash\left(U_{\varepsilon}^{(1)} \cup U_{\varepsilon}^{(2)}\right)} \frac{d k}{\omega_{\Lambda}^{(1)}(k)-\widetilde{\tau}_{\Lambda}^{\min }} .
$$

Thus, assertion (4.2) is proved.

We now analyse the functions $\varphi_{\Lambda}^{(\sigma)}(z)$. As stipulated, in the neighbourhood $U_{\varepsilon}^{(1)}$ we can perform a non-degenerate change of variables $k \rightarrow x(k), x\left(k_{1}^{\min }(\Lambda)\right)=0$ such that in $U_{\varepsilon}^{(1)}$ the function $\omega_{\Lambda}(k)$ will take the form

$$
\omega_{\Lambda}(k)=\tau_{\Lambda}^{(1)}+\frac{1}{2}\left(x_{1}^{2}+x_{2}^{2}\right) \beta,
$$

and the inverse map has the form

$$
k=k_{1}^{\min }(\Lambda)+W_{1} x+O\left(x^{2}\right) \equiv f_{1}^{\Lambda}(x), \quad x=\left(x_{1}, x_{2}\right) .
$$

Here $W_{1}=\left(\omega_{i j}^{\prime \prime}\left(k_{1}^{\min }(\Lambda)\right)\right)^{-1 / 2} \beta^{1 / 2}$ is a matrix that reduces the second differential of the function $\omega_{\Lambda}(k) \beta^{-1}$ at the point $k_{1}^{\min }(\Lambda)$ to a sum of squares; the variables $x_{1}, x_{2}$ run over the disc $x_{1}^{2}+x_{2}^{2}<2 \varepsilon$. The Jacobian $J_{\Lambda}^{(\sigma)}$ of the change of variable is a real analytic function in the $\operatorname{disc} x_{1}^{2}+x_{2}^{2}<2 \varepsilon$, and $J_{\Lambda}^{(\sigma)}(0)=\operatorname{det} W_{1}=\frac{1}{2 \pi} A_{\Lambda}^{(\sigma)} \beta$. It follows from (4.10) that

$$
\int_{U_{\varepsilon}^{1}} \frac{d k}{\omega_{\Lambda}(k)-z}=\int_{|x|^{2}<2 \varepsilon} \frac{J_{\Lambda}^{(1)}(x) d x}{\frac{1}{2} \beta\left(x_{1}^{2}+x_{2}^{2}\right)+\tau_{\Lambda}^{(1)}-z}=\frac{1}{\beta} \int_{|x|^{2}<2 \varepsilon} \frac{J_{\Lambda}^{(1)}(x) d x}{\frac{1}{2}|x|^{2}+\frac{\tau_{\Lambda}^{(1)}-z}{\beta}} .
$$

Using the results of [9] we find that for $z \in \mathcal{F}_{D}^{\Lambda}$,

$$
\int_{|x|^{2}<2 \varepsilon} \frac{J_{\Lambda}^{(1)}(x) d x}{\frac{1}{2}\left(x_{1}^{2}+x_{2}^{2}\right)+\frac{\tau_{\Lambda}^{(1)}-z}{\beta}}=h_{\Lambda}^{(1)}\left(\frac{\tau_{\Lambda}^{(1)}-z}{\beta}\right) \ln \left(\frac{\tau_{\Lambda}^{(1)}-z}{\beta}\right)+H_{\Lambda}^{(1)}\left(\frac{\tau_{\Lambda}^{(1)}-z}{\beta}\right),
$$

where $h_{\Lambda}^{(1)}(\omega)$ and $H_{\Lambda}^{(1)}(\omega)$ are analytic functions of the variable $\omega$ in the disc $|\omega|<\varepsilon$ that are uniformly bounded with respect to $\Lambda \in V_{\delta_{1} \beta^{2}}(\rho)$. Furthermore,

$$
h_{\Lambda}^{(1)}(0)=-2 \pi J_{\Lambda}^{(1)}(0)=-\beta A_{\Lambda}^{(1)} .
$$

Thus, (4.12)-(4.14) imply (4.4) for $\sigma=1$, where $K_{1}(\Lambda)=H_{\Lambda}^{1}(0)$,

$$
\begin{aligned}
& \Psi_{\Lambda}^{(1)}(z)=\frac{1}{\beta}\left[H_{\Lambda}^{(1)}\left(\frac{\tau_{\Lambda}^{(1)}-z}{\beta}\right)-H_{\Lambda}^{(1)}(0)\right], \\
& \Phi_{\Lambda}^{(1)}(z)=\frac{1}{\beta} \ln \left(\frac{\tau_{\Lambda}^{(1)}-z}{\beta}\right)\left[h_{\Lambda}^{(1)}\left(\frac{\tau_{\Lambda}^{(1)}-z}{\beta}\right)-h_{\Lambda}^{(1)}(0)\right] .
\end{aligned}
$$

The case of $\varphi_{\Lambda}^{(2)}(z)$ can be analysed in a similar fashion. Of course, here we assume $\beta$ to be so small that

$$
\left|\frac{\tau_{\Lambda}^{(1)}-z}{\beta}\right|<\varepsilon, \quad \sigma=1,2,
$$

for $z \in \mathcal{F}_{\Lambda}^{D}$. Lemma 4.1 is proved. 
4.3. The second and third terms of the series (4.1). We set

$$
I_{n}^{\Lambda}(z)=\int_{\mathbb{T}^{\nu}} \ldots \int_{\mathbb{T}^{\nu}} \frac{T_{n}^{\Lambda}\left(k_{1}, \ldots, k_{n} ; z\right)}{\prod_{i=1}^{n}\left(\omega_{\Lambda}\left(k_{i}\right)-z\right)} \prod_{i=1}^{n} d k_{i}
$$

First we derive a representation for $I_{2}^{\Lambda}(z)$ for $z \in \mathcal{F}_{D}^{\Lambda}$ and $\Lambda \in V_{\delta_{1} \beta^{2}}(\rho)$ (here $\rho \in \gamma_{\text {mult }}^{(2)}$ is a truncated double interval).

Lemma 4.2. Let $V_{\delta_{1} \beta^{2}}(\rho)$ be the $\delta_{1} \beta^{2}$-neighbourhood of an interval $\rho \subset \gamma_{\text {mult }}^{(2)}$ on a double curve $\gamma_{\text {mult }}^{(2)}$. For any $\Lambda \in V_{\delta_{1} \beta^{2}}(\rho)$ and $z \in \mathcal{F}_{D}^{\Lambda}$ we have the following representation for the integral $I_{2}^{\Lambda}(z)$ :

$$
I_{2}^{\Lambda}(z)=A_{\Lambda}^{(1)} A_{\Lambda}^{(2)} B_{\Lambda} \beta^{2} \ln \frac{\tau_{\Lambda}^{(1)}-z}{\beta} \ln \frac{\tau_{\Lambda}^{(2)}-z}{\beta}+G_{1}^{\Lambda} \ln \frac{\tau_{\Lambda}^{(1)}-z}{\beta}+G_{2}^{\Lambda} \ln \frac{\tau_{\Lambda}^{(2)}-z}{\beta}+\Psi_{\Lambda}(z)
$$

where $A_{1}$ and $A_{2}$ are the constants introduced in (4.4), the constant $B_{\Lambda}$ was introduced in (3.4), $G_{1}^{\Lambda}$ and $G_{2}^{\Lambda}$ are constants independent of $\beta, \Lambda$, and $z$, while $\Psi_{\Lambda}(z)$ is an analytic function in $\mathcal{F}_{D}^{\Lambda}$ satisfying the estimate

$$
\left|\Psi_{\Lambda}(z)\right|<C_{\Lambda}\left(\beta|\ln \beta|\left(\left|\ln \frac{\tau_{\Lambda}^{(1)}-z}{\beta}\right|+\left|\ln \frac{\tau_{\Lambda}^{(2)}-z}{\beta}\right|\right)+1\right)
$$

where the constant $C_{\Lambda}$ is independent of $\beta$ and $z$.

Proof of Lemma 4.2. We write

$$
\begin{aligned}
I_{2}^{\Lambda}(z)= & \int_{U_{\varepsilon}^{(1)}} d k_{1} \int_{U_{\varepsilon}^{(1)}} d k_{2} \frac{T_{2}^{\Lambda}\left(k_{1}, k_{2} ; z\right) d k_{1} d k_{2}}{\left(\omega_{\Lambda}\left(k_{1}\right)-z\right)\left(\omega_{\Lambda}\left(k_{2}\right)-z\right)} \\
& +\int_{U_{\varepsilon}^{(1)}} d k_{1} \int_{U_{\varepsilon}^{(2)}} d k_{2} \cdots+\int_{U_{\varepsilon}^{(2)}} d k_{1} \int_{U_{\varepsilon}^{(2)}} d k_{2} \cdots \\
& +\int_{U_{\varepsilon}^{(2)}} d k_{1} \int_{U_{\varepsilon}^{(1)}} d k_{2} \cdots+\int_{\left(U_{\varepsilon}^{(1)} \cup U_{\varepsilon}^{(2)}\right)^{\prime}} d k_{1} \int_{\left(U_{\varepsilon}^{(1)} \cup U_{\varepsilon}^{(2)}\right)^{\prime}} d k_{2} \cdots \\
& +\int_{U_{\varepsilon}^{(1)}} d k_{1} \int_{\left(U_{\varepsilon}^{(1)} \cup U_{\varepsilon}^{(2)}\right)^{\prime}} d k_{2} \cdots+\int_{U_{\varepsilon}^{(2)}} d k_{1} \int_{\left(U_{\varepsilon}^{(1)} \cup U_{\varepsilon}^{(2)}\right)^{\prime}} d k_{2} \cdots \\
& +\int_{\left(U_{\varepsilon}^{(1)} \cup U_{\varepsilon}^{(2)}\right)^{\prime}} d k_{1} \int_{U_{\varepsilon}^{(1)}} d k_{2} \cdots+\int_{\left(U_{\varepsilon}^{(1)} \cup U_{\varepsilon}^{(2)}\right)^{\prime}} d k_{1} \int_{U_{\varepsilon}^{(2)}} d k_{2} \cdots
\end{aligned}
$$

where

$$
\left(U_{\varepsilon}^{(1)} \cup U_{\varepsilon}^{(2)}\right)^{\prime}=\mathbb{T}^{2} \backslash\left(U_{\varepsilon}^{(1)} \cup U_{\varepsilon}^{(2)}\right)
$$

and the $U_{\varepsilon}^{\sigma}$ are the neighbourhoods of the points $k_{\sigma}^{\min }(\Lambda)$ introduced above.

First consider the first four integrals that have the form

$$
\int_{U_{\varepsilon}^{(\sigma)}} d k_{1} \int_{U_{\varepsilon}^{\left(\sigma^{\prime}\right)}} d k_{2} \cdots, \quad \sigma, \sigma^{\prime}=1,2 .
$$

In each neighbourhood $U_{\varepsilon}^{\sigma}$ we pass to the new coordinates $x^{\sigma}$ and let

$$
\widetilde{T}_{2, \sigma, \sigma^{\prime}}^{\Lambda}\left(x_{1}^{\sigma}, x_{2}^{\sigma^{\prime}} ; z\right)=T_{2}^{\Lambda}\left(f_{\sigma}^{\Lambda}\left(x_{1}^{\sigma}\right), f_{\sigma^{\prime}}^{\Lambda}\left(x_{2}^{\sigma^{\prime}}\right) ; z\right)
$$


where $f_{\sigma}^{\Lambda}$ is the map of the disc $\left\{\left|x^{\sigma}\right|^{2}<2 \varepsilon\right\}$ into the neighbourhood $U_{\varepsilon}^{\sigma}$ (see (4.11)). Then

$$
\begin{aligned}
I_{2 ; \sigma, \sigma^{\prime}}^{\Lambda}(z) & \equiv \int_{U_{\varepsilon}^{\sigma}} d k_{1} \int_{U_{\varepsilon}^{\sigma^{\prime}}} d k_{2} \frac{T_{2}^{\Lambda}\left(k_{1}, k_{2} ; z\right)}{\left(\omega_{\Lambda}\left(k_{1}\right)-z\right)\left(\omega_{\Lambda}\left(k_{2}\right)-z\right)} \\
& =\frac{1}{\beta^{2}} \int_{\left|x_{1}^{\sigma}\right|^{2}<2 \varepsilon} d x_{1}^{\sigma} \int_{\left|x_{2}^{\sigma^{\prime}}\right|^{2}<2 \varepsilon} d x_{2}^{\sigma^{\prime}} \frac{\widetilde{T}_{2, \sigma, \sigma^{\prime}}^{\Lambda}\left(x_{1}^{\sigma}, x_{2}^{\sigma^{\prime}} ; z\right) J_{\Lambda}^{\sigma}\left(x_{1}^{\sigma}\right) J_{\Lambda}^{\sigma^{\prime}}\left(x_{2}^{\sigma^{\prime}}\right)}{\left(\frac{1}{2}\left|x_{1}^{\sigma}\right|^{2}+\frac{\tau_{\Lambda}^{\sigma}-z}{\beta}\right)\left(\frac{1}{2}\left|x_{2}^{\sigma^{\prime}}\right|^{2}+\frac{\tau_{\Lambda}^{\sigma^{\prime}-z}}{\beta}\right)},
\end{aligned}
$$

where $J_{\Lambda}^{\sigma}\left(x_{1}^{\sigma}\right)$ is the Jacobian of the map $f_{\sigma}^{\Lambda}$. Next we apply formula (4.13) successively and obtain the following.

1. In the case $\sigma=\sigma^{\prime}$ we have

$$
\begin{aligned}
I_{2,(\sigma, \sigma)}^{\Lambda}=\frac{1}{\beta^{2}}[ & h_{\Lambda}^{\sigma}\left(\frac{\tau_{\Lambda}^{\sigma}-z}{\beta} ; z\right)\left(\ln \frac{\tau_{\Lambda}^{\sigma}-z}{\beta}\right)^{2} \\
& \left.+g_{\Lambda}^{\sigma}\left(\frac{\tau_{\Lambda}^{\sigma}-z}{\beta} ; z\right) \ln \frac{\tau_{\Lambda}^{\sigma}-z}{\beta}+H_{\Lambda}^{\sigma}\left(\frac{\tau_{\Lambda}^{\sigma}-z}{\beta} ; z\right)\right] .
\end{aligned}
$$

2. In the case $\sigma \neq \sigma^{\prime}$ we have $I_{2, \sigma, \sigma^{\prime}}^{\Lambda}=I_{2, \sigma^{\prime}, \sigma}^{\Lambda}=I_{2,(1,2)}^{\Lambda}$ (since the function $T_{2}^{\Lambda}\left(k_{1}, k_{2} ; z\right)$ is symmetric with respect to the variables $\left.k_{1}, k_{2}\right)$ and

$$
\begin{aligned}
I_{2,\left(\sigma, \sigma^{\prime}\right)}^{\Lambda}=\frac{1}{\beta^{2}}[ & h_{\Lambda}^{\sigma, \sigma^{\prime}}\left(\frac{\tau_{\Lambda}^{\sigma}-z}{\beta}, \frac{\tau_{\Lambda}^{\sigma^{\prime}}-z}{\beta} ; z\right) \ln \frac{\tau_{\Lambda}^{\sigma}-z}{\beta} \ln \frac{\tau_{\Lambda}^{\sigma^{\prime}}-z}{\beta} \\
& +g_{\Lambda, 1}^{\sigma, \sigma^{\prime}}\left(\frac{\tau_{\Lambda}^{\sigma}-z}{\beta}, \frac{\tau_{\Lambda}^{\sigma^{\prime}}-z}{\beta} ; z\right) \ln \frac{\tau_{\Lambda}^{1}-z}{\beta} \\
& +g_{\Lambda, 2}^{\sigma, \sigma^{\prime}}\left(\frac{\tau_{\Lambda}^{\sigma}-z}{\beta}, \frac{\tau_{\Lambda}^{\sigma^{\prime}}-z}{\beta} ; z\right) \ln \frac{\tau_{\Lambda}^{2}-z}{\beta} \\
& \left.+H_{\Lambda}^{\sigma, \sigma^{\prime}}\left(\frac{\tau_{\Lambda}^{\sigma}-z}{\beta}, \frac{\tau_{\Lambda}^{\sigma^{\prime}}-z}{\beta} ; z\right)\right] .
\end{aligned}
$$

In the expansions (4.18) and (4.19), the functions

$$
h_{\Lambda}^{\sigma}(\omega, z), \quad h_{\Lambda}^{\sigma, \sigma^{\prime}}\left(\omega_{1}, \omega_{2} ; z\right), \quad g_{\Lambda}^{\sigma}(\omega ; z), \quad g_{\Lambda, i}^{\sigma, \sigma^{\prime}}\left(\omega_{1}, \omega_{2} ; z\right), \quad i=1,2,
$$

and

$$
H_{\Lambda}^{\sigma}(\omega, z), \quad H_{\Lambda}^{\sigma, \sigma^{\prime}}\left(\omega_{1}, \omega_{2} ; z\right)
$$

are analytic functions of $(\omega, z)$ or $\left(\omega_{1}, \omega_{2}, z\right)$, in the domains $\left\{|\omega|<\varepsilon, z \in \mathcal{F}_{D}^{\Lambda}\right\}$ or $\left\{\left|\omega_{1}\right|<\varepsilon,\left|\omega_{2}\right|<\varepsilon, z \in \mathcal{F}_{D}^{\Lambda}\right\}$, respectively. Next, it follows from the definition of $T_{2}^{\Lambda}\left(k_{1}, k_{2} ; z\right)$ that

$$
T_{2}^{\Lambda}(k, k, z)=0
$$

for all $z \in \mathbb{C}$ and all $k \in \mathbb{T}^{2}$, and for $z \in \mathcal{F}_{\Lambda}^{D}$ we have

$$
\left|T_{2}^{\Lambda}\left(k_{1}, k_{2} ; z\right)-T_{2}^{\Lambda}\left(k_{1}, k_{2} ; \tau_{\Lambda}^{\sigma}\right)\right|<C \beta^{4},
$$

where $C$ is a constant independent of $\Lambda, k_{1}, k_{2}, z$, and $\beta$. Furthermore, for $\sigma=\sigma^{\prime}$,

$$
h_{\Lambda}^{\sigma}(0, z)=\beta^{2}\left(A_{\Lambda}^{\sigma}\right)^{2} T_{2}^{\Lambda}\left(k_{\min }^{\sigma}, k_{\min }^{\sigma} ; z\right)=0
$$

and, consequently,

$$
h_{\Lambda}^{\sigma}(\omega, z)=\omega r_{\Lambda}^{\sigma}(\omega ; z),
$$

where $r_{\Lambda}^{\sigma}(\omega, z)$ is an analytic function in the domain $\left\{|\omega|<\varepsilon, z \in \mathcal{F}_{D}^{\Lambda}\right\}$ satisfying the estimate

$$
\left|r_{\Lambda}^{\sigma}(\omega, z)\right|<\operatorname{const} \beta^{2}
$$


where const is independent of $\omega, z$, and $\beta$. By (4.20),

$$
g_{\Lambda}^{\sigma}(0, z)=g_{\Lambda}^{\sigma}\left(0, \tau_{\Lambda}^{\sigma}\right)+O\left(\beta^{4}\right),
$$

while

$$
\frac{1}{\beta^{2}} g_{\Lambda}^{\sigma}\left(0, \tau_{\Lambda}^{\sigma}\right)=G_{\Lambda}^{\sigma}+O(\beta)
$$

where $G_{\Lambda}^{\sigma}$ is a constant independent of $\beta$.

Since $\omega \ln \omega$ and $\omega|\ln \omega|^{2}$ are bounded functions for $|\omega|<\varepsilon$, and $\left|H_{\Lambda}^{\sigma}(\omega ; z)\right|<\operatorname{const} \beta^{2}$, where const is independent of $\omega, z$, and $\beta$, we obtain from the above that

$$
I_{2, \sigma, \sigma}^{\Lambda}(z)=G_{\Lambda}^{\sigma} \ln \frac{\tau_{\Lambda}^{\sigma}-z}{\beta}+\Psi_{\Lambda}^{\sigma}(z), \quad \sigma=1,2
$$

where $\Psi_{\Lambda}^{\sigma}$ is an analytic function of $z \in \mathcal{F}_{D}^{\Lambda}$ satisfying the estimate

$$
\left|\Psi_{\Lambda}^{\sigma}(z)\right|<C\left(\beta\left|\ln \frac{\tau_{\Lambda}^{\sigma}-z}{\beta}\right|+1\right)
$$

and the constant $C$ is independent of $\beta, \Lambda$, and $z$.

We now consider the case $\sigma \neq \sigma^{\prime}$ and again write down the expansions

$$
\begin{aligned}
& h_{\Lambda}^{\sigma, \sigma^{\prime}}\left(\omega_{1}, \omega_{2} ; z\right)=h_{\Lambda}^{\sigma, \sigma^{\prime}}(0,0 ; z)+\omega_{1} r_{\Lambda, 1}^{\sigma, \sigma^{\prime}}\left(\omega_{1}, \omega_{2} ; z\right)+\omega_{2} r_{\Lambda, 2}^{\sigma, \sigma^{\prime}}\left(\omega_{1}, \omega_{2} ; z\right), \\
& g_{\Lambda, i}^{\sigma, \sigma^{\prime}}\left(\omega_{1}, \omega_{2} ; z\right)=g_{\Lambda, i}^{\sigma, \sigma^{\prime}}(0,0 ; z)+\omega_{1} p_{\Lambda, i}^{\sigma, \sigma^{\prime},(1)}\left(\omega_{1}, \omega_{2} ; z\right)+\omega_{2} p_{\Lambda, i}^{\sigma, \sigma^{\prime},(2)}\left(\omega_{1}, \omega_{2} ; z\right),
\end{aligned}
$$

where $r_{\Lambda, i}^{\sigma, \sigma^{\prime}}, p_{\Lambda, i}^{\sigma, \sigma^{\prime},(j)}, i, j=1,2$, are analytic functions of $\omega_{1}, \omega_{2}$ and $z$ in the domain $\left\{\left|\omega_{1}\right|<\varepsilon,\left|\omega_{2}\right|<\varepsilon, z \in \mathcal{F}_{D}^{\Lambda}\right\}$. Furthermore, $\left|r_{\Lambda, i}^{\sigma, \sigma^{\prime}}\right|<C \beta^{2}, i=1,2$, and $\left|p_{\Lambda, i}^{\sigma, \sigma^{\prime},(j)}\right|<C \beta^{2}$, $i, j=1,2$, where $C$ is a constant independent of $\omega_{1}, \omega_{2}, z$ and $\beta$. Hence, using the estimate

$$
\max _{0<\omega<D \beta} \omega|\ln \omega|<\bar{D} \beta|\ln \beta|,
$$

where $\bar{D}$ is a constant, we conclude that

$$
\begin{aligned}
\frac{1}{\beta^{2}} \mid\left(\frac{\tau_{\Lambda}^{1}-z}{\beta} r_{\Lambda, 1}^{\sigma, \sigma^{\prime}}+\frac{\tau_{\Lambda}^{2}-z}{\beta} r_{\Lambda, 2}^{\sigma, \sigma^{\prime}}\right) \ln \frac{\tau_{\Lambda}^{1}-z}{\beta} & \ln \frac{\tau_{\Lambda}^{2}-z}{\beta} \mid \\
& <C \beta|\ln \beta|\left(\left|\ln \frac{\tau_{\Lambda}^{1}-z}{\beta}\right|+\left|\ln \frac{\tau_{\Lambda}^{2}-z}{\beta}\right|\right),
\end{aligned}
$$

where $C$ is a constant independent of $z$ and $\beta$.

Similar arguments show that

$$
\frac{1}{\beta^{2}}\left|\sum_{i, j=1,2} \frac{\tau_{\Lambda}^{(j)}-z}{\beta} \ln \frac{\tau_{\Lambda}^{(i)}-z}{\beta} p_{\Lambda, i}^{\sigma, \sigma^{\prime}, j}\right|<C\left(\beta\left(\left|\ln \frac{\tau_{\Lambda}^{(1)}-z}{\beta}\right|+\left|\ln \frac{\tau_{\Lambda}^{(2)}-z}{\beta}\right|\right)+1\right) .
$$

Using the representations

$$
\begin{aligned}
h_{\Lambda}^{\sigma, \sigma^{\prime}}(0,0, z) & =h_{\Lambda}^{\sigma, \sigma^{\prime}}\left(0,0, \tau_{\Lambda}^{\min }\right)+O\left(\beta^{4}\right) \\
g_{\Lambda, i}^{\sigma, \sigma}(0,0, z) & =g_{\Lambda, i}^{\sigma, \sigma^{\prime}}\left(0,0, \tau_{\Lambda}^{\min }\right)+O\left(\beta^{4}\right) \\
\frac{1}{\beta^{2}} h_{\Lambda}^{\sigma, \sigma^{\prime}}\left(0,0, \tau_{\Lambda}^{\min }\right) & =A_{1} A_{2} T_{2}^{\Lambda}\left(k_{\min }^{\sigma}, k_{\min }^{\sigma^{\prime}}, \tau_{\Lambda}^{\min }\right)=A_{1} A_{2} \beta^{2} B_{\Lambda} \\
\frac{1}{\beta^{2}} g_{\Lambda, i}^{\sigma, \sigma^{\prime}}\left(0,0, \tau_{\Lambda}^{\min }\right) & =G_{\Lambda, i}^{\sigma, \sigma^{\prime}}+O(\beta)
\end{aligned}
$$


where $A_{1}$ and $A_{2}$ are the constants introduced in (4.3), the $B_{\Lambda}$ are the quantities introduced in (3.12) and (3.13), and the $G_{\Lambda, i}^{\sigma, \sigma}$ are constants independent of $\beta$, we finally obtain that for $\sigma \neq \sigma^{\prime}$,

$$
\begin{aligned}
I_{2,\left(\sigma, \sigma^{\prime}\right)}^{\Lambda}(z)= & A_{1} A_{2} \beta^{2} B_{\Lambda} \ln \frac{\tau_{\Lambda}^{(1)}-z}{\beta} \ln \frac{\tau_{\Lambda}^{(2)}-z}{\beta} \\
& +G_{\Lambda, 1}^{\sigma, \sigma^{\prime}} \ln \frac{\tau_{\Lambda}^{(1)}-z}{\beta}+G_{\Lambda, 2}^{\sigma, \sigma^{\prime}} \ln \frac{\tau_{\Lambda}^{(2)}-z}{\beta}+\Psi_{\Lambda}^{\sigma, \sigma^{\prime}}(z),
\end{aligned}
$$

where $\Psi_{\Lambda}^{\sigma, \sigma^{\prime}}(z)$ is an analytic function in $\mathcal{F}_{D}^{\Lambda}$ satisfying the estimate

$$
\left|\Psi_{\Lambda}^{\sigma, \sigma^{\prime}}(z)\right|<C\left(\beta|\ln \beta|\left(\left|\ln \frac{\tau_{\Lambda}^{(1)}-z}{\beta}\right|+\left|\ln \frac{\tau_{\Lambda}^{(2)}-z}{\beta}\right|\right)+1\right),
$$

where $C$ is a constant independent of $z$ and $\beta$.

We now consider the integral

$$
I_{2,(3,3)}^{\Lambda} \equiv \int_{\left(U_{\varepsilon}^{(1)} \cup U_{\varepsilon}^{(2)}\right)^{\prime}} d k_{1} \int_{\left(U_{\varepsilon}^{(1)} \cup U_{\varepsilon}^{(2)}\right)^{\prime}} d k_{2} \cdots .
$$

Using the arguments we used in the proof of formula (4.9), together with the estimate (3.3), we find that this integral is a bounded analytic function:

$$
\left|I_{2,(3,3)}^{\Lambda}(z)\right|<\bar{C}
$$

where $\bar{C}$ is a constant independent of $z, \beta$, and $\Lambda$.

Finally, consider the integral

$$
I_{2(1,3)}^{\Lambda}(z)=\int_{U_{\varepsilon}^{(1)}} \frac{d k_{1}}{\omega_{\Lambda}\left(k_{1}\right)-z} \int_{\left(U_{\varepsilon}^{(1)} \cup U_{\varepsilon}^{(2)}\right)^{\prime}} \frac{T_{2}^{\Lambda}\left(k_{1}, k_{2}, z\right) d k_{2}}{\omega_{\Lambda}\left(k_{1}\right)-z} .
$$

Similarly to the way we derived formula (4.9), we obtain that the inner integral in (4.28) is equal to

$$
\int_{\left(U_{\varepsilon}^{(1)} \cup U_{\varepsilon}^{(2)}\right)^{\prime}} \frac{T_{2}^{\Lambda}\left(k_{1}, k_{2}, z\right) d k_{2}}{\omega_{\Lambda}\left(k_{2}\right)-z}=K_{\Lambda}\left(k_{1} ; z\right),
$$

where $K_{\Lambda}\left(k_{1}, z\right), k_{1} \in \mathbb{T}^{2}$, is an analytic function of $z \in \mathcal{F}_{D}^{\Lambda}$ that is infinitely smooth with respect to the variable $k_{1}$. Next, passing to the outer integral with respect to the variable $k_{1}$ and again using formula (4.13), we obtain the representation

$$
I_{2(1,3)}(z)=h_{\Lambda}^{(1,3)}\left(\frac{\tau_{\Lambda}^{(1)}-z}{\beta} ; z\right) \ln \frac{\tau_{\Lambda}^{(1)}-z}{\beta}+H_{\Lambda}^{(1,3)}\left(\frac{\tau_{\Lambda}^{(1)}-z}{\beta} ; z\right),
$$

where $h_{\Lambda}^{(1,3)}(\omega, z)$ and $H_{\Lambda}^{(1,3)}(\omega, z)$ are analytic functions of the variables $\omega, z$ in the domain $\left\{|\omega|<\varepsilon, z \in \mathcal{F}_{D}^{\Lambda}\right\}$.

Again arguing as above we have

$$
I_{2,(1,3)}^{\Lambda}(z)=I_{2,(3,1)}^{\Lambda}(z)=G_{\Lambda}^{(1,3)} \ln \frac{\tau_{\Lambda}^{(1)}-z}{\beta}+\Psi_{\Lambda}^{(1,3)}(z)
$$

where $G_{\Lambda}^{(1,3)}$ is a constant independent of $\beta$ and $z$, while $\Psi_{\Lambda}^{(1,3)}(z)$ is an analytic function of $z \in \mathcal{F}_{D}^{\Lambda}$ satisfying the estimate

$$
\left|\Psi_{\Lambda}^{(1,3)}(z)\right|<C\left(\beta\left|\ln \frac{\tau_{\Lambda}^{(1)}-z}{\beta}\right|+1\right),
$$

where $C>0$ is a constant independent of $z$ and $\beta$. The remaining two integrals are dealt with in a similar fashion, yielding representations similar to (4.29). Taking the 
sum of the representations (4.23), (4.26), (4.27), (4.29) we arrive at the expansion (4.16). Lemma 4.2 is proved.

By repeating the arguments used in the proof of Lemma 4.2, applying the estimate

$$
\left|T_{3}^{\Lambda}\left(k_{1}, k_{2}, k_{3} ; z\right)-T_{3}^{\Lambda}\left(k_{1}, k_{2}, k_{3} ; \tau_{\Lambda}^{\sigma}\right)\right|<\operatorname{const} \beta^{6}
$$

and observing that

$$
T_{3}^{\Lambda}\left(k_{1}, k_{2}, k_{3} ; z\right)=0 \quad \text { for } \quad k_{i}=k_{j}, \quad i \neq j, \quad i, j=1,2,3,
$$

we can derive the following representation for $I_{3}^{\Lambda}(z)$ :

$I_{3}^{\Lambda}(z)=A_{1} A_{2} \widehat{B}_{\Lambda} \beta^{4} \ln \frac{\tau_{\Lambda}^{(1)}-z}{\beta} \ln \frac{\tau_{\Lambda}^{(2)}-z}{\beta}+\beta^{2} L_{1}^{\Lambda} \ln \frac{\tau_{\Lambda}^{(1)}-z}{\beta}+\beta^{2} L_{2}^{\Lambda} \ln \frac{\tau_{\Lambda}^{(2)}-z}{\beta}+\Psi_{\Lambda}^{(3)}(z)$

with $\widehat{B}_{\Lambda}=\widehat{B}_{\Lambda}^{0}+O(\beta)$, where $\widehat{B}_{\Lambda}^{0}$ is a constant independent of $\beta, \quad L_{i}^{\Lambda}=L_{i, 0}^{\Lambda}+O(\beta)$ with the $L_{i, 0}^{\Lambda}$ being constants also independent of $\beta$, and $\Psi_{\Lambda}^{3}(z)$ is an analytic function of $z \in \mathcal{F}_{D}^{\Lambda}$ satisfying the estimate

$$
\left|\Psi_{\Lambda}^{(3)}(z)\right|<C \beta^{2}\left\{\beta|\ln \beta|\left(\left|\ln \frac{\tau_{\Lambda}^{(1)}-z}{\beta}\right|+\left|\ln \frac{\tau_{\Lambda}^{(2)}-z}{\beta}\right|\right)+1\right\}
$$

where $C$ is a constant independent of $\beta$ and $z$.

4.4. Tail of the series (4.1). Now we estimate the integrals $I_{n}^{\Lambda}(z), n>3$. First we state a general estimate for the function $T_{n}^{\Lambda}$, whose proof can be found in [2]

Theorem 4.1. For any $z \in \mathcal{F}_{D}^{\Lambda}$ and any set $\gamma_{1}, \ldots, \gamma_{k}$ of pairwise disjoint subsets of the set of indices $\{1, \ldots, n\},\left|\gamma_{i}\right| \geq 2, i=1, \ldots, k$, the estimate

$$
\left|T_{n}^{\Lambda}\left(k_{1}, \ldots, k_{n} ; z\right)\right|<A\left(C \beta^{2}\right)^{n-1} n^{n / 2} \prod_{i=1}^{k} \prod_{j \in \gamma_{i}, j \neq j_{i}}\left(k_{j_{i}}-k_{j}\right)^{2}
$$

holds, where $j_{i} \in \gamma_{i}$ is some index in the set $\gamma_{i}$, and $A, C$ are constants independent of $z$, $n, \Lambda$, and $\beta$.

Lemma 4.3. For $z \in \mathcal{F}_{D}^{\Lambda}$ and $n>3$ we have the estimate

$\left|I_{n}^{\Lambda}(z)\right|<\bar{A}(\bar{C} \beta)^{n-2} n^{n / 2}\left(\left|\ln \frac{\tau_{\Lambda}^{(1)}-z}{\beta}\right|\left|\ln \frac{\tau_{\Lambda}^{(2)}-z}{\beta}\right|+\left|\ln \frac{\tau_{\Lambda}^{(1)}-z}{\beta}\right|+\left|\ln \frac{\tau_{\Lambda}^{(2)}-z}{\beta}\right|+1\right)$,

where $\bar{A}$ and $\bar{C}$ are positive constants independent of $z, \Lambda, n$, and $\beta$.

Corollary. It follows from the estimate (4.32) that

$$
\left|\sum_{n=4}^{\infty} I_{n}^{\Lambda}(z) \frac{1}{n !}\right|<C \beta^{2}\left(\left|\ln \frac{\tau_{\Lambda}^{(1)}-z}{\beta}\right|\left|\ln \frac{\tau_{\Lambda}^{(2)}-z}{\beta}\right|+\left|\ln \frac{\tau_{\Lambda}^{(1)}-z}{\beta}\right|+\left|\ln \frac{\tau_{\Lambda}^{(2)}-z}{\beta}\right|+1\right)
$$

where $C$ is a constant independent of $z, \Lambda$, and $\beta$.

Proof of Lemma 4.3. Let $\gamma_{1}, \gamma_{2} \subseteq\{1, \ldots, n\}$ be two disjoint subsets of indices. We denote by $\mathcal{H}_{\gamma_{1}, \gamma_{2}} \subset\left(\mathbb{T}^{2}\right)^{n}$ the domain defined by the following condition:

$\mathcal{H}_{\gamma_{1}, \gamma_{2}}=\left\{\left(k_{1}, \ldots, k_{n}\right): k_{i} \in U_{\varepsilon}^{(1)}, i \in \gamma_{1} ; k_{j} \in U_{\varepsilon}^{(2)}, j \in \gamma_{2} ; k_{s} \in \mathbb{T}^{2} \backslash U_{\varepsilon}^{(1)} \cup U_{\varepsilon}^{(2)}, s \notin \gamma_{1} \cup \gamma_{2}\right\}$.

We will estimate the integral

$$
\int_{\mathcal{H}_{\gamma_{1}, \gamma_{2}}} \frac{T_{n}^{\Lambda}\left(k_{1}, \ldots, k_{n} ; z\right)}{\prod\left(\omega_{\Lambda}\left(k_{i}\right)-z\right)} \prod d k_{i}
$$


when $\left|\gamma_{1}\right| \geq 2,\left|\gamma_{2}\right| \geq 2$. In each of the sets $\left\{k_{i}, i \in \gamma_{1}\right\}$ and $\left\{k_{j}, j \in \gamma_{2}\right\}$ we choose the points $k_{i_{1}}$ and $k_{j_{1}}$ that are closest to $k_{1}^{\min }(\Lambda)$ and $k_{2}^{\min }(\Lambda)$, respectively, and apply (4.31):

$$
\begin{aligned}
& \left|T_{n}^{\Lambda}\left(k_{1}, \ldots, k_{n} ; z\right)\right| \\
& \quad<A\left(C \beta^{2}\right)^{n-1} n^{n / 2} \prod_{i \in \gamma_{1}, i \neq i_{1}}\left(k_{i_{1}}-k_{i}\right)^{2} \prod_{j \in \gamma_{2}, j \neq j_{1}}\left(k_{j_{1}}-k_{j}\right)^{2} \\
& \quad<A\left(C \beta^{2}\right)^{n-1} n^{n / 2} 2^{p_{1}+p_{2}} \prod_{i \in \gamma_{1}, i \neq i_{1}}\left(\Delta_{i_{1}}^{2}+\Delta_{i}^{2}\right) \prod_{j \in \gamma_{2}, j \neq j_{1}}\left(\Delta_{j_{1}}^{2}+\Delta_{j}^{2}\right),
\end{aligned}
$$

where $\Delta_{i}=\left(k_{i}-k_{1}^{\min }(\Lambda)\right), \quad \Delta_{j}=\left(k_{j}-k_{2}^{\min }(\Lambda)\right)$, and $p_{m}=\left|\gamma_{m}\right|, m=1,2$. Since $\left|\Delta_{i_{1}}\right| \leq \Delta_{i}$ and $\left|\Delta_{j_{1}}\right| \leq \Delta_{j}$, we obviously have the inequalities

$$
\begin{gathered}
\prod_{i \in \gamma_{1}, i \neq i_{1}}\left(\Delta_{i_{1}}^{2}+\Delta_{i}^{2}\right) \leq 2^{p_{1}-1} \sum_{i_{0} \in \gamma_{1}} \prod_{i \in \gamma_{1}, i \neq i_{0}} \Delta_{i}^{2}, \\
\prod_{j \in \gamma_{2}, j \neq j_{1}}\left(\Delta_{j_{1}}^{2}+\Delta_{j}^{2}\right) \leq 2^{p_{2}-1} \sum_{j_{0} \in \gamma_{2}} \prod_{j \in \gamma_{2}, j \neq j_{0}} \Delta_{j}^{2} .
\end{gathered}
$$

From this and (4.33) we obtain that

$$
\begin{aligned}
\int_{\mathcal{H}_{\gamma_{1}, \gamma_{2}}} & \frac{T_{n}^{\Lambda}\left(k_{1}, \ldots, k_{n} ; z\right)}{\prod\left(\omega_{\Lambda}\left(k_{i}\right)-z\right)} \prod d k_{i} \\
< & A(C \beta)^{2 n-2} n^{n / 2} 4^{p_{1}+p_{2}} p_{1} p_{2}\left(\int_{U_{\varepsilon}^{(1)}} \frac{\Delta^{2} d k}{\left|\omega_{\Lambda}(k)-z\right|}\right)^{p_{1}-1} \\
& \times\left(\int_{U_{\varepsilon}^{(2)}} \frac{\Delta^{2} d k}{\left|\omega_{\Lambda}(k)-z\right|}\right)^{p_{2}-1} \int_{U_{\varepsilon}^{(1)}} \frac{d k}{\left|\omega_{\Lambda}(k)-z\right|} \\
& \times \int_{U_{\varepsilon}^{(2)}} \frac{d k}{\left|\omega_{\Lambda}(k)-z\right|}\left(\int_{\left(U_{\varepsilon}^{(1)} \cup U_{\varepsilon}^{(2)}\right)^{\prime}} \frac{d k}{\left|\omega_{\Lambda}(k)-z\right|}\right)^{n-\left(p_{1}+p_{2}\right)} \\
< & A\left(C \beta^{2}\right)^{n-1} n^{n / 2} 4^{p_{1}+p_{2}} p_{1} p_{2}\left(\frac{M}{\beta}\right)^{n-2} \widehat{\varphi}_{1}(z) \widehat{\varphi}_{2}(z),
\end{aligned}
$$

where

$$
\begin{array}{r}
M=\max \left\{\sup _{z \in \mathcal{F}_{D}^{\Lambda}} \beta \int_{U_{\varepsilon}^{(1)}} \frac{\left(k-k_{\min }^{(1)}(\Lambda)\right)^{2} d k}{\left|\omega_{\Lambda}(k)-z\right|}, \sup _{z \in \mathcal{F}_{D}^{\Lambda}} \beta \int_{U_{\varepsilon}^{(2)}} \frac{\left(k-k_{\min }^{(2)}(\Lambda)\right)^{2} d k}{\left|\omega_{\Lambda}(k)-z\right|},\right. \\
\left.\sup _{z \in \mathcal{F}_{D}^{\Lambda}} \beta \int_{\left(U_{\varepsilon}^{(1)} \cup U_{\varepsilon}^{(2)}\right)^{\prime}} \frac{d k}{\left|\omega_{\Lambda}(k)-z\right|}\right\}
\end{array}
$$

and

$$
\widehat{\varphi}_{i}(z)=\int_{U_{\varepsilon}^{(i)}} \frac{d k}{\left|\omega_{\Lambda}(k)-z\right|}, \quad i=1,2 .
$$

A similar estimate is obtained when one of the sets $\gamma_{i}$ consists of a single element and the other is non-empty. In the case where one of the sets is empty, the integral over the set $\mathcal{H}_{\gamma_{1}, \gamma_{2}}$ is no greater than

$$
A\left(C \beta^{2}\right)^{n-1} n^{n / 2}\left(\frac{M}{\beta}\right)^{n-1} p_{i} \widehat{\varphi}_{\Lambda}^{(i)}(z) 4^{p_{i}}
$$


where $i$ is the index in the non-empty set. Finally, when both sets are empty, $\gamma_{1}=\gamma_{2}=\varnothing$, the integrals do not exceed

$$
A\left(C \beta^{2}\right)^{(n-1)} n^{n / 2}\left(\frac{M}{\beta}\right)^{n} .
$$

Next, it is easy to calculate that

$$
\sum_{\substack{\gamma_{1}, \gamma_{2}: \gamma_{i} \in\{1, \ldots, n\} \\ i=1,2, \gamma_{1} \cap \gamma_{2}=\varnothing}} 4^{\left|\gamma_{1}\right|+\left|\gamma_{2}\right|}\left|\gamma_{1}\right| \cdot\left|\gamma_{2}\right|<9^{n} n^{2} \text { and } \sum_{\substack{\gamma \in\{1, \ldots, n\}, \gamma \neq \varnothing\\}} 4^{|\gamma|}|\gamma|<n \cdot 5^{n} .
$$

We now estimate $\widehat{\varphi}_{i}(z)$. Since

$$
\left|\omega_{\Lambda}(k)-z\right|>\frac{1}{\sqrt{2}}\left(\omega_{\Lambda}(k)-u+|v|\right), \quad u=\operatorname{Re} z, \quad v=\operatorname{Im} z
$$

for $z \in \mathcal{F}_{D}^{\Lambda}$, using (4.12), (4.13) we obtain

$$
\begin{aligned}
\beta \widehat{\varphi}_{1}(z) & <\beta \sqrt{2} \int \frac{d k}{\omega_{\Lambda}(k)-u+|v|}<C\left(-\ln \frac{\tau_{\Lambda}^{(1)}-u+|v|}{\beta}+1\right) \\
& <C\left(-\ln \left|\frac{\tau_{\Lambda}^{(1)}-z}{\beta}\right|+1\right)<\bar{C}\left(\left|\ln \frac{\tau_{\Lambda}^{(1)}-z}{\beta}\right|+1\right),
\end{aligned}
$$

where $C>0$ is a constant independent of $\beta$ and $z$. From (4.35) (4.39) we obtain (4.32). Lemma 4.3 is proved.

4.5. The final representation of $\Delta_{\Lambda}(z)$. Collecting together all the representations and estimates obtained above we arrive at the following assertion.

Theorem 4.2. For $\Lambda$ in the $V_{\delta_{1} \beta^{2}}$-neighbourhood of a truncated double interval $\rho \subset \gamma_{\text {mult }}^{(2)}$ and for $z \in \mathcal{F}_{D}^{\Lambda}$, the Fredholm determinant $\Delta_{\Lambda}(z)$ introduced in (4.1) admits the following representation:

$$
\Delta_{\Lambda}(z)=\Delta_{\Lambda}^{0}(z)+\Psi_{\Lambda}^{(1)}(z)+\Psi_{\Lambda}^{(2)}(z)
$$

where

$$
\Delta_{\Lambda}^{0}(z)=-\frac{\widetilde{a}_{1}^{\Lambda}}{\beta} \ln \frac{\tau_{\Lambda}^{(1)}-z}{\beta}-\frac{\widetilde{a}_{2}^{\Lambda}}{\beta} \ln \frac{\tau_{\Lambda}^{(2)}-z}{\beta}+\widetilde{B}_{\Lambda} \widetilde{a}_{1}^{\Lambda} \widetilde{a}_{2}^{\Lambda} \ln \frac{\tau_{\Lambda}^{(1)}-z}{\beta} \ln \frac{\tau_{\Lambda}^{(2)}-z}{\beta}+\frac{K_{1}}{\beta} .
$$

Here,

$$
\widetilde{a}_{i}^{\Lambda}=\widetilde{a}_{i, 0}^{\Lambda}+O(\beta), \quad i=1,2,
$$

and

$$
\widetilde{B}_{\Lambda}=B_{\Lambda, 0}+O(\beta)
$$

where $\widetilde{a}_{i, 0}^{\Lambda}$ and $B_{\Lambda, 0}$ are constants independent of $\beta$ and $z$.

In (4.40), $\Psi_{\Lambda}^{(1)}(z)$ and $\Psi_{\Lambda}^{(2)}(z)$ are analytic functions in the domain $\mathcal{F}_{D}^{\Lambda}$ admitting the estimates

$$
\left|\Psi_{\Lambda}^{(1)}(z)\right|<C\left[\beta^{2}\left|\ln \frac{\tau_{\Lambda}^{(1)}-z}{\beta} \ln \frac{\tau_{\Lambda}^{(2)}-z}{\beta}\right|+\beta|\ln \beta|\left(\left|\ln \frac{\tau_{\Lambda}^{(1)}-z}{\beta}\right|+\left|\ln \frac{\tau_{\Lambda}^{(2)}-z}{\beta}\right|\right)+1\right]
$$

and

$$
\left|\Psi_{\Lambda}^{(2)}(z)\right|<C\left(\frac{\left|\tau_{\Lambda}^{(1)}-z\right|}{\beta^{2}}\left|\ln \frac{\tau_{\Lambda}^{(1)}-z}{\beta}\right|+\frac{\left|\tau_{\Lambda}^{(2)}-z\right|}{\beta^{2}}\left|\ln \frac{\tau_{\Lambda}^{(2)}-z}{\beta}\right|\right)
$$

where $C$ is a constant independent of $\beta, z$ and $\Lambda$. 
4.6. Adjacent levels (zeros of $\Delta_{\Lambda}(z)$ in the interval $0<\tau_{\Lambda}^{\min }-z<D \beta^{2}$ ). The case $B_{\Lambda}>0$ (non-threshold interval $\rho$ ). For $z \in\left[\tau_{\Lambda}^{\min }-D \beta^{2}, \tau_{\Lambda}^{\min }\right]$, the first two summands in (4.41) are positive and are greater than

$$
\frac{\text { const }}{\beta}|\ln \beta D|
$$

consequently, one half their sum majorises the sum of two terms in $\Delta_{\Lambda}(z)$,

$$
\frac{K(\Lambda)}{\beta}+\Psi_{\Lambda}^{(2)}(z)
$$

The remaining half of their sum, together with the term

$$
\widetilde{B}_{\Lambda} \widetilde{a}_{1}^{\Lambda} \widetilde{a}_{2}^{\Lambda} \ln \frac{\tau_{\Lambda}^{(1)}-z}{\beta} \ln \frac{\tau_{\Lambda}^{(2)}-z}{\beta}>0,
$$

majorises $\Psi_{\Lambda}^{(1)}(z)$. Thus, on the interval indicated, for $\Lambda \in V_{\delta_{1} \beta^{2}}(\rho)$ the function $\Delta_{\Lambda}(z)$ is greater than 0 and, consequently, $\mathcal{T}_{\Lambda}$ has no adjacent levels. Thus, we have proved the second assertion of Theorem 3.1

4.7. The case $B_{\Lambda}<0$ (threshold interval $\rho$ ). Zeros of the function $\Delta_{\Lambda}^{0}(z)$ in the domain $\mathcal{F}_{\Lambda}^{D}$. We introduce the quantities

$$
\begin{aligned}
& r_{\max }(\rho)=\max \left\{\sup _{\Lambda \in V_{\delta_{1} \beta^{2}}(\rho)} \widetilde{a}_{1}^{\Lambda}\left|\widetilde{B}_{\Lambda}\right|, \sup _{\Lambda \in V_{\delta_{1} \beta^{2}}(\rho)} \widetilde{a}_{2}^{\Lambda}\left|\widetilde{B}_{\Lambda}\right|\right\}, \\
& r_{\min }(\rho)=\min \left\{\inf _{\Lambda \in V_{\delta_{1} \beta^{2}}(\rho)} \widetilde{a}_{1}^{\Lambda}\left|\widetilde{B}_{\Lambda}\right|, \inf _{\Lambda \in V_{\delta_{1} \beta^{2}}(\rho)} \widetilde{a}_{2}^{\Lambda}\left|\widetilde{B}_{\Lambda}\right|\right\} .
\end{aligned}
$$

Lemma 4.4. In the case $\Lambda \in V_{\delta_{1} \beta^{2}}(\rho)$, where $\rho$ is a threshold interval, the function $\Delta_{\Lambda}^{0}(z)$ has no zeros in $\mathcal{F}_{\Lambda}^{D}$ if

$$
d(\Lambda, \rho)>\frac{1}{\kappa_{2}} e^{-\frac{1}{r_{\max }(\rho) \beta}},
$$

and has exactly one zero $z_{0}^{\Lambda}=\varepsilon(\Lambda)$ in this domain if

$$
d(\Lambda, \rho)<\frac{1}{\kappa_{1}} e^{-\frac{1}{r_{\min }(\rho) \beta}} .
$$

This zero is real and simple and satisfies the estimate

$$
0<\tau_{\Lambda}^{\min }-z_{0}^{\Lambda}<\beta \exp \left\{-\frac{\widetilde{a}_{1}^{\Lambda}+\widetilde{a}_{2}^{\Lambda}}{\widetilde{a}_{1}^{\Lambda} \widetilde{a}_{2}^{\Lambda}\left|\widetilde{B}_{\Lambda}\right| \beta}\right\}\left(1+O\left(\beta^{2}\right)\right) .
$$

Here $d(\Lambda, \rho)$ is the distance from $\Lambda$ to the interval $\rho$, and the constants $\kappa_{1}, \kappa_{2}$ were introduced in (3.6).

Proof. We introduce the quantities

$$
u=\widetilde{a}_{1} \ln \frac{\tau_{\Lambda}^{(1)}-z}{\beta}<0, \quad v=\widetilde{a}_{2} \ln \frac{\tau_{\Lambda}^{(2)}-z}{\beta}<0,
$$

and assume for definiteness that $\tau_{2}^{\Lambda} \geq \tau_{1}^{\Lambda}=\tau_{\Lambda}^{\min }$. Then the equation $\Delta_{\Lambda}^{0}(z)=0$ (for real $z$ ) is equivalent to the system of equations

$$
\left\{\begin{array}{l}
-\frac{1}{\beta}(u+v)+\widetilde{B}_{\Lambda} u v+\frac{K_{\Lambda}}{\beta}=0, \\
\beta\left(e^{v / \widetilde{a}_{2}^{\Lambda}}-e^{u / \widetilde{a}_{1}^{\Lambda}}\right)=\tau_{2}-\tau_{1} .
\end{array}\right.
$$


The first equation is the equation of the lower branch of the hyperbola

$$
\left(u-\frac{1}{\widetilde{B}_{\Lambda} \beta}\right)\left(v-\frac{1}{\widetilde{B}_{\Lambda} \beta}\right)=\frac{1}{\widetilde{B}_{\Lambda}^{2} \beta^{2}}-\frac{K_{\Lambda}}{\widetilde{B}_{\Lambda} \beta}, \quad u, v<0
$$

with the asymptotes

$$
u=\frac{1}{\widetilde{B}_{\Lambda} \beta}<0, \quad v=\frac{1}{\widetilde{B}_{\Lambda} \beta}<0
$$

the second equation represents the graph of the function

$$
V(u)=\widetilde{a}_{2}^{\Lambda} \ln \left(\frac{\tau_{\Lambda}^{(2)}-\tau_{\Lambda}^{(1)}}{\beta}+e^{u / \widetilde{a}_{1}}\right),
$$

which tends monotonically to the limit

$$
\lim _{u \rightarrow-\infty} V(u)=\widetilde{a}_{2}^{\Lambda} \ln \frac{\tau_{\Lambda}^{(2)}-\tau_{\Lambda}^{(1)}}{\beta}
$$

as $u \rightarrow-\infty$ and satisfies

$$
V(u)>\widetilde{a}_{2} \ln \frac{\tau_{\Lambda}^{(2)}-\tau_{\Lambda}^{(1)}}{\beta} .
$$

Thus, a negative solution $\left(u_{0}<0, v_{0}<0\right)$ of the system (4.47) exists if the horizontal asymptote of the hyperbola is situated above the horizontal asymptote of the curve $V(u)$, that is,

$$
\frac{1}{\widetilde{B}_{\Lambda} \beta}>\widetilde{a}_{2}^{\Lambda} \ln \frac{\tau_{\Lambda}^{(2)}-\tau_{\Lambda}^{(1)}}{\beta} .
$$

In the case where the opposite inequality holds, there are no negative solutions $\left(u_{0}<0\right.$, $\left.v_{0}<0\right)$ of the system (4.47). The arrangement of the hyperbola and the curve (4.50) (when there exists a solution of the system (4.47)) is depicted in Figure 4.

Since the point of intersection $\left(u_{0}, v_{0}\right)$ of the hyperbola (4.48) and the curve (4.50) with $u_{0}<0$ and $v_{0}<0$, lies to the left of the vertical asymptote of the hyperbola, that is, $u_{0}<\frac{1}{\widetilde{B}_{\Lambda} \beta}$, we find that a root $z_{0}^{(1)}$ of the equation $\Delta_{\Lambda}^{0}(z)=0$ satisfies the inequality

$$
\left|\tau_{\Lambda}^{\min }-z_{0}^{(1)}\right|<\beta \exp \left\{-\frac{1}{\left|\widetilde{B}_{\Lambda}\right| \beta \widetilde{a}_{1}^{\Lambda}}\right\}
$$

and, consequently, belongs to the domain $\mathcal{F}_{D}^{\Lambda}$ for sufficiently small $\beta$. Apart from the solution $\left(u_{0}, v_{0}\right)=\left(u_{0}^{(1)}, v_{0}^{(1)}\right)$, the system (4.47) has another solution $\left(u_{0}^{(2)}, v_{0}^{(2)}\right)$, which corresponds to a root $z_{0}^{(2)}$ of the equation $\Delta_{\Lambda}^{(0)}=0$ lying outside the domain $\mathcal{F}_{D}^{\Lambda}$. Indeed, we have

$$
V(0)=\ln \left(\frac{\tau_{\Lambda}^{(2)}-\tau_{\Lambda}^{(1)}}{\beta}+1\right)=O\left(\beta^{2}\right)>0
$$

as follows from (3.7). Next, we observe that the ordinate of the intersection of the second branch of the hyperbola with the $v$-axis is equal to $K_{\Lambda}$. If $K_{\Lambda}>0$, then $K_{\Lambda}>V(0)$ for sufficiently small $\beta$, since $K_{\Lambda}$ is independent of $\beta$, and $u_{0}^{(2)}>0$ and $v_{0}^{(2)}>0$, as is clear from Figure 4. Hence, $\tau_{\Lambda}^{\min }-z_{0}^{(2)}>\beta>D \beta^{2}$. If $K_{\Lambda} \leq 0$, then $u_{0}^{(2)}>K_{\Lambda}$, as is again clear from the picture, that is, again

$$
\tau_{(1)}^{\Lambda}-z_{0}^{2}>\beta e^{K(\Lambda) / \widetilde{a}_{1}^{\Lambda}}>D \beta^{2}
$$

for sufficiently small $\beta$. 


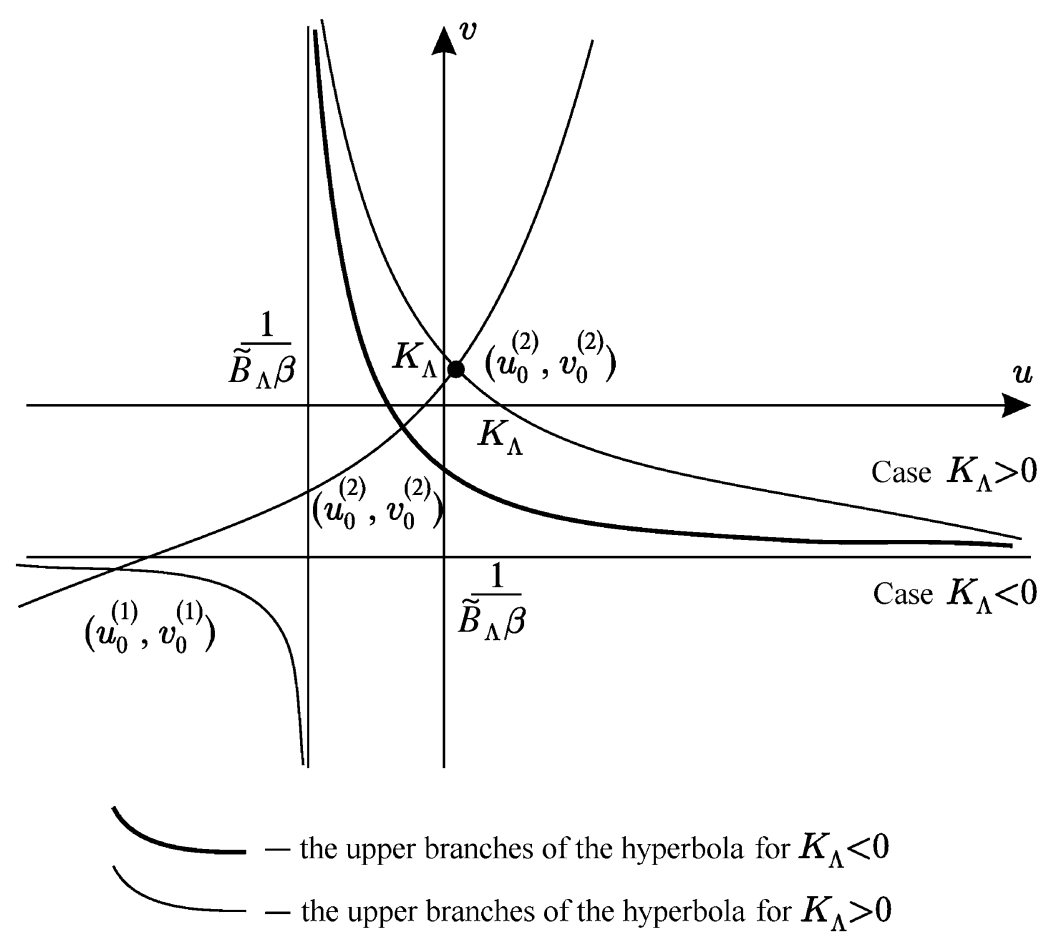

Figure 4. The arrangement of the branches of the hyperbola and the graph of $V(u)$

Thus, we have shown that in the domain $\mathcal{F}_{D}^{\Lambda}$, provided (4.52) holds, there is exactly one real root of $\bar{\Delta}_{\Lambda}^{0}(z)$, and there are no real roots if this condition is violated.

We observe that by (3.6) for

$$
d(\Lambda, \rho)<\frac{1}{\kappa_{1}} \exp \left\{-\frac{1}{\widetilde{a}_{2}^{\Lambda}\left|\widetilde{B}_{\Lambda}\right| \beta}\right\},
$$

condition (4.52) holds, while for

$$
d(\Lambda, \rho)>\frac{1}{\kappa_{2} \beta} \exp \left\{-\frac{1}{\widetilde{a}_{2}^{\Lambda}\left|\widetilde{B}_{\Lambda}\right| \beta}\right\}
$$

it is violated. On the other hand, (4.44) implies (4.55), and (4.45) implies (4.54). Next, from the second equation in (4.47), it is evident that $v_{0}^{(1)} / \widetilde{a}_{2}>u_{0}^{(1)} / \widetilde{a}_{1}$, that is, the point $\left(u_{0}^{(1)}, v_{0}^{(1)}\right)$ lies above the straight line $v=\frac{\widetilde{a}_{2}}{\widetilde{a}_{1}} u$. The coordinates of the point of intersection of this straight line with the lower branch of the hyperbola (4.48) are

$$
\bar{u}_{0}^{(1)}=-\frac{\widetilde{a}_{1}^{\Lambda}+\widetilde{a}_{2}^{\Lambda}}{\left|\widetilde{B}_{\Lambda}\right| \widetilde{a}_{2}^{\Lambda} \beta}\left(1+O\left(\beta^{2}\right)\right), \quad \bar{v}_{0}^{(1)}=-\frac{\widetilde{a}_{1}^{\Lambda}+\widetilde{a}_{2}^{\Lambda}}{\left|\widetilde{B}_{\Lambda}\right| \widetilde{a}_{1}^{\Lambda} \beta}\left(1+O\left(\beta^{2}\right)\right) .
$$

This fact and the inequality $u_{0}^{(1)}<\bar{u}_{0}^{(1)}$ imply inequality (4.46).

We now show that $z_{0}^{(1)}$ is a simple zero. Indeed, it is easy to verify that

$$
\left.\left(\Delta_{\Lambda}\right)^{\prime}\right|_{\bar{z}=z_{0}^{(1)}}=\frac{\widetilde{a}_{1}^{\Lambda}}{\beta} \frac{1}{\tau_{\Lambda}^{(1)}-z_{0}^{(1)}}+\frac{\widetilde{a}_{2}^{\Lambda}}{\beta} \frac{1}{\tau_{\Lambda}^{(2)}-z_{0}^{(1)}}-\widetilde{B}_{\Lambda} \widetilde{a}_{2}^{\Lambda} u_{0} \frac{1}{\tau_{\Lambda}^{(2)}-z_{0}^{(1)}}-\widetilde{B}_{\Lambda} \widetilde{a}_{1}^{\Lambda} v_{0} \frac{1}{\tau_{\Lambda}^{(1)}-z_{0}^{(1)}},
$$


where $u_{0}$ and $v_{0}$ solve the system (4.47). We observe that the straight line in the $(u, v)$ plane,

$$
\frac{\widetilde{B}_{\Lambda} \widetilde{a}_{2}^{\Lambda}}{\tau_{\Lambda}^{(2)}-z_{0}^{(1)}} u+\frac{\widetilde{B}_{\Lambda} \widetilde{a}_{1}^{\Lambda}}{\tau_{\Lambda}^{(1)}-z_{0}^{(1)}} v-\frac{\widetilde{a}_{1}^{\Lambda}}{\beta} \frac{1}{\tau_{\Lambda}^{(1)}-z_{0}^{(1)}}-\frac{\widetilde{a}_{2}^{\Lambda}}{\beta} \frac{1}{\tau_{\Lambda}^{(2)}-z_{0}^{(1)}}=0
$$

passes through $\left(u=\frac{1}{\tilde{B}_{\Lambda} \beta}, v=\frac{1}{\tilde{B}_{\Lambda} \beta}\right)$, which is the point of intersection of the asymptotes of the hyperbola (4.48), which contains the point $\left(u_{0}, v_{0}\right)$, and the straight line (4.57) lies in the second and fourth quadrants formed by the asymptotes (4.49). Thus, the straight line (4.57) does not intersect the hyperbola (4.48) and, consequently, the right-hand side of the expression (4.56) is not equal to zero: thus, $\left(\Delta_{\Lambda}^{0}\right)_{z=z_{0}^{(1)}}^{\prime} \neq 0$, that is, $z_{0}^{(1)}$ is a simple root.

We now show that in the domain $\mathcal{F}_{\Lambda}^{D}$ there are no other (complex) zeros apart from $z_{0}^{(1)}$. For $z \in \mathcal{F}_{\Lambda}^{D}$, setting

$$
\begin{aligned}
\widehat{u} & =\widetilde{a}_{1}^{\Lambda} \ln \left|\frac{\tau_{\Lambda}^{(1)}-z}{\beta}\right|, & \widehat{v} & =\widetilde{a}_{2}^{\Lambda} \ln \left|\frac{\tau_{\Lambda}^{(2)}-z}{\beta}\right|, \\
\theta_{1} & =\arg \frac{\tau_{\Lambda}^{(1)}-z}{\beta}, & \theta_{2} & =\arg \frac{\tau_{\Lambda}^{(2)}-z}{\beta},
\end{aligned}
$$

we can easily see that the equation $\Delta_{\Lambda}(z)=0$ implies the following two equations:

$$
\operatorname{Re} \Delta_{\Lambda}^{0}(z) \equiv-\frac{1}{\beta}(\widehat{u}+\widehat{v})+\widetilde{B}_{\Lambda} \widehat{u} \widehat{v}-\widetilde{B}_{\Lambda} \widetilde{a}_{1}^{\Lambda} \widetilde{a}_{2}^{\Lambda} \theta_{1} \theta_{2}+\frac{K(\Lambda)}{\beta}=0, \quad \widehat{u}, \widehat{v}<0,
$$

and

$$
\operatorname{Im} \Delta_{\Lambda}^{0}(z) \equiv-\frac{\widetilde{a}_{1}^{\Lambda}}{\beta} \theta_{1}-\frac{\widetilde{a}_{2}^{\Lambda}}{\beta} \theta_{2}+\widetilde{B}_{\Lambda} \widetilde{a}_{1}^{\Lambda} \theta_{1} \widehat{v}+\widetilde{B}_{\Lambda} \widetilde{a}_{2}^{\Lambda} \theta_{2} \widehat{u}=0 .
$$

For fixed $\theta_{1}$ and $\theta_{2}$, the first equation is again the equation of the lower branch of a hyperbola on the $(\widehat{u}, \widehat{v})$-plane with the asymptotes

$$
\widehat{u}=\frac{1}{\beta \widetilde{B}_{\Lambda}}, \quad \widehat{v}=\frac{1}{\beta \widetilde{B}_{\Lambda}},
$$

and the second equation is the equation of a straight line passing through the intersection point of the asymptotes and lying in the second and fourth quadrants (since $\theta_{1}$ and $\theta_{2}$ have the same signs and do not vanish simultaneously for complex $z$ ). Thus, since there does not exist a pair of numbers $(\widehat{u}, \widehat{v})$ which simultaneously satisfies both equations, the function $\Delta_{\Lambda}^{0}$ also has no complex $\left(\operatorname{Im} z_{0} \neq 0\right)$ zeros $z_{0}$. Lemma 4.4 is proved.

\subsection{The zeros of the Fredholm determinant in $\mathcal{F}_{D}^{\Lambda}$.}

Lemma 4.5. When

$$
\tau_{\Lambda}^{(2)}-\tau_{\Lambda}^{(1)}>3 \beta \exp \left\{-\frac{1}{\widetilde{a}_{2}^{\Lambda}\left|\widetilde{B}_{\Lambda}\right| \beta}\right\},
$$

the full Fredholm determinant $\Delta_{\Lambda}(z)$ does not vanish in the domain $\mathcal{F}_{D}^{\Lambda}$, that is, the operator $\mathcal{T}_{\Lambda}$ has no adjacent levels below the continuous spectrum.

When

$$
\tau_{\Lambda}^{(2)}-\tau_{\Lambda}^{(1)}<\beta \exp \left\{-\frac{2}{\widetilde{a}_{2}^{\Lambda}\left|\widetilde{B}_{\Lambda}\right| \beta}-\frac{K(\Lambda)+2}{2 \widetilde{a}_{2}^{\Lambda}}-1\right\}
$$

with $K(\Lambda)$ being the constant occurring in the expansion (4.2), the Fredholm determinant has only one zero in the domain $\mathcal{F}_{\Lambda}^{D}$ and this zero is real. Thus, under condition (4.60) the operator $\mathcal{T}_{\Lambda}$ has only one adjacent level below the continuous spectrum. 
Corollary. Note that by (3.6), inequality (4.59) implies the estimate

$$
d(\Lambda, \rho)>\frac{3}{\kappa_{2}} \exp \left\{-\frac{1}{\widetilde{a}_{2}^{\Lambda}\left|\widetilde{B}_{\Lambda}\right| \beta}\right\}>\frac{1}{k_{2}} e^{-\frac{1}{r_{\max }(\rho) \beta}}
$$

and inequality (4.60) implies that

$$
d(\Lambda, \rho)<\frac{1}{\kappa_{1}} \exp \left\{-\frac{1}{\widetilde{a}_{2}^{\Lambda}\left|\widetilde{B}_{\Lambda}\right| \beta}\right\}<\frac{1}{k_{1}} e^{-\frac{1}{r_{\min }(\rho) \beta}}
$$

which coincides with the assertions in the first part of Theorem 3.1.

Proof of Lemma 4.5. Let $\Gamma$ be the contour that forms the boundary of the domain $\mathcal{F}_{D}^{\Lambda}$; this contour consists of two parts: $\Gamma_{1}$, which is the half-circle $\left|\tau_{\Lambda}^{\min }-z\right|=D \beta^{2}$, $\operatorname{Re}\left(\tau_{\Lambda}^{\min }-z\right) \geq 0$, and $\Gamma_{2}$, a segment on the imaginary axis $-D \beta^{2} \leq \operatorname{Im}\left(\tau_{\Lambda}^{\min }-z\right) \leq D \beta^{2}$.

We shall show that in both cases (4.59) and (4.60) $)$ the inequality

$$
\left|\Delta_{\Lambda}-\Delta_{\Lambda}^{0}\right|<\left|\Delta_{\Lambda}^{0}\right|
$$

holds on the whole contour $\Gamma$, whence the assertion of Lemma 4.5 will follow by Rouché's theorem [10] and Lemma 4.4 .

1. Let $z \in \Gamma_{1}$. Since $\left(\right.$ for $\tau_{\Lambda}^{(1)}=\tau_{\Lambda}^{(\min )}$ )

$$
\left|\frac{\tau_{\Lambda}^{(2)}-z}{\beta}\right|<\left|\frac{\tau_{\Lambda}^{(1)}-z}{\beta}\right|+\left|\frac{\tau_{\Lambda}^{(2)}-\tau_{\Lambda}^{(1)}}{\beta}\right|=D \beta+O\left(\beta^{2}\right),
$$

the leading term in $\Delta_{\Lambda}^{(0)}$ is the sum of the first two terms, which is of the order of $-\frac{\ln \beta}{\beta}$. The other summands in $\Delta_{\Lambda}^{0}$ are of order $O\left((\ln \beta)^{2}\right)$ and $O(1 / \beta)$. Thus, for sufficiently small $\beta$,

$$
\left|\Delta_{\Lambda}^{0}(z)\right|>C \frac{|\ln \beta|}{\beta}, \quad z \in \Gamma_{1},
$$

where $C>0$ is a constant independent of $\beta$. As follows from the estimates (4.42), (4.43), the functions $\Psi_{\Lambda}^{(1)}$ and $\Psi_{\Lambda}^{(2)}$ on the contour $\Gamma$ do not exceed

$$
\left|\Psi_{\Lambda}^{(1)}(z)\right|<\text { const, } \quad\left|\Psi_{\Lambda}^{(2)}\right|<\text { const }|\ln \beta| .
$$

From (4.64) and (4.65) we obtain (4.63).

2. We now consider the case of inequality (4.59) and $z \in \Gamma_{2}$, that is, $z=\tau_{\Lambda}^{(1)}+i t$, where $|t|<D \beta^{2}$. We analyse the case where $t$ only runs over one half of this segment: $0 \leq t \leq D \beta^{2}$; the case of negative $t$ can be considered in a similar fashion. We introduce the following notation (see 4.58) :

$$
u=\widetilde{a}_{1}^{\Lambda} \ln \left|\frac{\tau_{\Lambda}^{(1)}-z}{\beta}\right|=\widetilde{a}_{1}^{\Lambda} \ln \frac{t}{\beta}, \quad-\infty<u<\widetilde{a}_{1}^{\Lambda} \ln D \beta=u_{0}<0,
$$

$$
\begin{gathered}
v=\widetilde{a}_{2}^{\Lambda} \ln \left|\frac{\tau_{\Lambda}^{(2)}-z}{\beta}\right|=\widetilde{a}_{2}^{\Lambda} \ln \frac{\sqrt{t^{2}+\left(\tau_{\Lambda}^{(2)}-\tau_{\Lambda}^{(1)}\right)^{2}}}{\beta} \\
\widetilde{a}_{2}^{\Lambda} \ln \frac{\tau_{\Lambda}^{(2)}-\tau_{\Lambda}^{(1)}}{\beta}<v<\widetilde{a}_{2}^{\Lambda} \ln \frac{\sqrt{D^{2} \beta^{2}+\left(\tau_{\Lambda}^{(2)}-\tau_{\Lambda}^{(1)}\right)^{2}}}{\beta}=v_{0}<\widetilde{a}_{2}^{\Lambda} \ln 2 D \beta<0
\end{gathered}
$$


where $|u|>|v|$. It is obvious that then

$$
\begin{aligned}
\operatorname{Re} \Delta_{\Lambda}^{0} & =-\frac{1}{\beta}(u+v)+\widetilde{B}_{\Lambda} u v+\widetilde{B}_{\Lambda} \widetilde{a}_{1}^{\Lambda} \widetilde{a}_{2}^{\Lambda} \frac{\pi}{2} \theta(t)+\frac{K(\Lambda)}{\beta} \\
& >-\frac{1}{\beta}(u+v)+\widetilde{B}_{\Lambda} u v+\frac{K(\Lambda)}{\beta}, \\
\operatorname{Im} \Delta_{\Lambda}^{0} & =\frac{\widetilde{a}_{1}^{\Lambda}}{\beta} \frac{\pi}{2}+\frac{\widetilde{a}_{2}^{\Lambda}}{\beta}|\theta(t)|-\widetilde{B}_{\Lambda} \widetilde{a}_{2}^{\Lambda} u|\theta(t)|-\widetilde{B}_{\Lambda} \widetilde{a}_{1}^{\Lambda} v \frac{\pi}{2},
\end{aligned}
$$

where

$$
\theta(t)=\arg \frac{\left(\tau_{\Lambda}^{(2)}-z\right)}{\beta}=-\arctan \frac{t}{\left|\tau_{\Lambda}^{(2)}-\tau_{\Lambda}^{(1)}\right|}<0 .
$$

Furthermore, for $z=\tau_{\Lambda}^{(1)}+i t$, the functions $\Psi_{\Lambda}^{(1)}(z)$ and $\Psi_{\Lambda}^{(2)}(z),|t|<D \beta$, admit the estimates

$$
\begin{aligned}
& \left|\Psi_{\Lambda}^{(1)}(z)\right|<\bar{C}\left(\beta^{2} u v-\beta u-\beta v+1\right), \\
& \left|\Psi_{\Lambda}^{(2)}(z)\right|<\bar{C}\left(-\frac{u}{\beta} e^{u / \widetilde{a}_{1}^{\Lambda}}-\frac{v}{\beta} e^{v / \widetilde{a}_{2}^{\Lambda}}\right),
\end{aligned}
$$

which follow from (4.42), (4.43). The second estimate implies that for $u<\widetilde{a}_{1}^{\Lambda} \ln D \beta$ and $v<\widetilde{a}_{2}^{\Lambda} \ln 2 D \beta$ and for sufficiently small $\beta$, we have

$$
\left|\Psi_{\Lambda}^{(2)}(z)\right|<-\widetilde{C}(u+v) .
$$

Here $\bar{C}, \widetilde{C}$ are constants independent of $\beta, \Lambda, u, v$. Furthermore, $u$ and $v$ are connected by the relation

$$
\beta^{2}\left(e^{2 v / \widetilde{a}_{2}^{\Lambda}}-e^{2 u / \widetilde{a}_{1}^{\Lambda}}\right)=\left(\tau_{\Lambda}^{(2)}-\tau_{\Lambda}^{(1)}\right)^{2} .
$$

Using this relation to express $v$ in terms of $u$, we obtain the monotonic function

$$
\widetilde{V}(u)=\frac{\widetilde{a}_{2}^{\Lambda}}{2} \ln \left[\frac{\left(\tau_{\Lambda}^{(2)}-\tau_{\Lambda}^{(1)}\right)^{2}}{\beta^{2}}+e^{2 u / \widetilde{a}_{1}^{\Lambda}}\right],
$$

defined on the segment $-\infty<u<\widetilde{a}_{1}^{\Lambda} \ln D \beta$. We denote the graph of this function by $\mathcal{J}$. Thus, as $t$ varies from $D \beta^{2}$ to zero, the point $(u, v)$ moves along the curve $\mathcal{J}$ from the point $\left(u_{0}, v_{0}=V\left(v_{0}\right)\right)$ so that the value $\widetilde{V}(u)$ approaches $\widetilde{V}(-\infty)=\widetilde{a}_{2}^{\Lambda} \ln \frac{\tau_{\Lambda}^{(2)}-\tau_{\Lambda}^{(1)}}{\beta}$ monotonically as $u \rightarrow-\infty$ (see (4.51)).

Next, condition (4.59) means that

$$
\widetilde{V}(-\infty)>\ln 3+\frac{1}{\beta \widetilde{B}_{\Lambda}}>1+\frac{1}{\widetilde{B}_{\Lambda} \beta},
$$

and, consequently, our curve $\mathcal{J}$ lies in the domain

$$
S_{1}=\left\{(u, v): v>\frac{1}{\beta \widetilde{B}_{\Lambda}}+1,|u|>|v|, u<a_{1} \ln D \beta<0, v<a_{2} \ln 2 D \beta<0\right\} .
$$

Some simple calculations using (4.42), (4.43), and (4.67) show that in this domain the inequality

$$
\operatorname{Re} \Delta_{\Lambda}^{0}>C\left(\beta^{2} u v-\beta(u+v)+1\right)>\left|\Psi_{\Lambda}^{(1)}(z)+\Psi_{\Lambda}^{(2)}(z)\right|
$$

holds, so that

$$
\left|\Delta_{\Lambda}^{0}(z)\right|>\left|\Delta_{\Lambda}(z)-\Delta_{\Lambda}^{0}(z)\right|
$$


for all $z \in \Gamma_{2}$. It is evident from inequality (4.67) that to prove inequality (4.69) it is sufficient to prove that in the domain $S_{1}$ the estimate

$$
L_{1}(u, v) \equiv-\frac{1}{\beta}(u+v)+\widetilde{B}_{\Lambda} u v+\frac{K(\Lambda)}{\beta}>C|\ln \beta|\left(\beta^{2} u v-\beta(u+v)+1\right) \equiv O(u, v)
$$

holds, that is, $L_{1}(u, v)-O(u, v)>0$.

It is easy to see that this inequality is valid in the domain $G_{1}$ bounded from below by the lower branch of the hyperbola $\mathcal{H}_{+}: L_{1}(u, v)-O(u, v)=0$, and from above and from the sides by the negative coordinate half-axes, and, possibly, by a segment of the upper branch of the hyperbola $\mathcal{H}_{+}$(at a finite distance from the origin; see Figure 5).

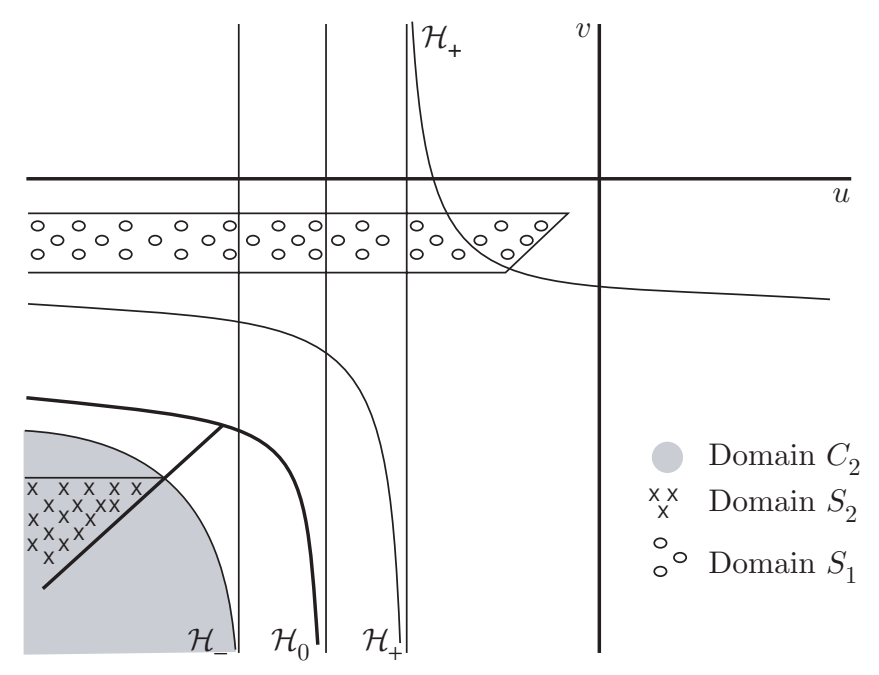

Figure 5. Arrangement of the domains $G_{1}, G_{2}, S_{1}, S_{2}$ and the hyperbolas $\mathcal{H}_{-}, \mathcal{H}_{0}, \mathcal{H}_{+}$

Since the horizontal asymptote of the hyperbola $\mathcal{H}_{+}$is the straight line

$$
v=\frac{1}{\beta \bar{B}}+O(\beta)
$$

(situated below the straight line $v=\frac{1}{\bar{\beta} B}+1$ ), the domain $S_{1}$ is contained in $G_{1}$ and therefore inequality (4.71) holds for all points $(u, v) \in S_{1}$. Thus, using (4.61), Lemma4.4 and the estimates (4.63) and (4.70), the first assertion of Lemma 4.5) is proved.

We now consider the case of inequality (4.60). Here we obviously have (see (4.51))

$$
\widetilde{V}(-\infty)<\frac{2}{\widetilde{B}_{\Lambda} \beta}-\frac{1}{2}|K(\Lambda)|-2 .
$$

As we shall now show, in the domain

$$
S_{2}=\left\{(u, v): v<\frac{2}{\beta \widetilde{B}_{\Lambda}}-\frac{1}{2}|K(\Lambda)|-1, u, v<0,|u|>|v|\right\}
$$

the inequality

$$
\left|-\frac{1}{\beta}(u+v)+\widetilde{B}_{\Lambda} u v+\frac{K(\Lambda)}{\beta}\right|-r>C|\ln \beta|\left(\beta^{2} u v-\beta(u+v)+1\right)
$$


holds, where

$$
r=\left|\widetilde{B}_{\Lambda} \widetilde{a}_{1}^{\Lambda} \widetilde{a}_{2}^{\Lambda}\left(\frac{\pi}{2}\right)^{2}\right|
$$

This now implies that

$$
\left|\operatorname{Re} \Delta_{0}(z)\right|>\left|\Psi_{\Lambda}^{(1)}(z)+\right| \Psi_{\Lambda}^{(2)}(z)|| \quad \text { or } \quad\left|\Delta_{\Lambda}^{0}(z)\right|>\left|\Delta_{\Lambda}(z)-\Delta_{\Lambda}^{0}(z)\right| .
$$

Since the domain $S_{2}$ lies below the hyperbola $\mathcal{H}_{0}: L_{1}(u, v)=0$, the estimate (4.72) is equivalent to the inequality

$$
-L_{1}(u, v)-O(u, v)-r>0,
$$

which holds in the domain $G_{2}$, which is bounded from above by the hyperbola

$$
\mathcal{H}_{-}:-L_{1}(u, v)-O(u, v)-r=0 .
$$

This hyperbola lies below the hyperbola $H_{0}$; its horizontal asymptote is given by the equation

$$
v=\frac{1}{\beta \widetilde{B}_{\Lambda}}+O(\beta),
$$

and $(\bar{u}, \bar{v})$, the point of intersection with the straight line $\bar{u}=\bar{v}$ is

$$
\bar{u}=\bar{v}=\frac{2}{\beta B}-\frac{K(\Lambda)}{2}+O(\beta) .
$$

Thus, the straight line bounding the domain $S_{2}$ from above lies below the horizontal straight line passing through the point $(\bar{u}, \bar{v})$, that is, $S_{2} \subset G_{2}$ and inequality (4.73) holds at points of the domain $S_{2}$.

Note that we have actually proved that

$$
\left|\operatorname{Re} \Delta_{\Lambda}^{0}(z)\right|>O(u, v)
$$

in the domains $G_{1}$ and $G_{2}$. We now show that in the gap between these domains lying in the half-plane $|u|>|v|$, that is, between the lower branches of the hyperbolas $\mathcal{H}_{+}$ and $\mathcal{H}_{-}$and above the straight line $u=v$ (we denote this gap by $S_{3}$ ), the estimate

$$
\left|\Delta_{\Lambda}^{0}(z)\right|>O(u, v)>\left|\Delta_{\Lambda}(z)-\Delta_{\Lambda}^{0}(z)\right|
$$

holds. First we observe that

$$
\left|\tau_{\Lambda}^{(2)}-z\right|>\beta \exp \left\{-\frac{2}{\beta\left|\widetilde{B}_{\Lambda}\right| \widetilde{a}_{2}}-\frac{1}{2} \frac{K(\Lambda)}{\widetilde{a}_{2}}-\frac{1}{\widetilde{a}_{2}}\right\},
$$

for the points on the curve $\mathcal{J}$ that lie above the horizontal line $v=\frac{2}{\beta \widetilde{B}_{\Lambda}}-\frac{1}{2} K(\Lambda)-1$. At the same time, $\tau_{\Lambda}^{(2)}-\tau_{\Lambda}^{(1)}$ satisfies the estimate (4.60) and therefore

$$
\frac{\tau_{\Lambda}^{(2)}-\tau_{\Lambda}^{(1)}}{\left|\tau_{\Lambda}^{(2)}-z\right|}=\cos \theta(t)<e^{-1 / 2}=\arccos \theta_{0}, \quad \text { where } \quad \theta_{0}>\frac{\pi}{6} ;
$$

in other words, the angle takes values satisfying $|\theta(t)|>\pi / 6$ for the points of that part of the curve $\mathcal{J}$ that lies above the indicated straight line. To derive the estimate (4.74) we use the inequality

$$
\left|\Delta_{\Lambda}^{0}(z)\right|>\frac{1}{2}\left(\left|\operatorname{Re} \Delta_{\Lambda}^{0}(z)\right|+\left|\operatorname{Im} \Delta_{\Lambda}^{0}(z)\right|\right) .
$$

We observe that the linear form

$$
\operatorname{Im} \Delta_{\Lambda}^{0}(z)=\frac{1}{\beta}\left(\frac{\pi \widetilde{a}_{1}^{\Lambda}}{2}+\widetilde{a}_{2}^{\Lambda}|\theta|\right)-\widetilde{B}_{\Lambda} u \widetilde{a}_{2}^{\Lambda}|\theta|-\widetilde{B}_{\Lambda} v \widetilde{a}_{1}^{\Lambda} \frac{\pi}{2}
$$


is negative below the hyperbola $\mathcal{H}_{+}$for any $\theta$. Thus, in the requisite domain,

$$
\begin{aligned}
\left|\operatorname{Im} \Delta_{\Lambda}^{0}(z)\right| & =-\frac{1}{\beta}\left(\frac{\pi}{2} \widetilde{a}_{1}^{\Lambda}+\widetilde{a}_{2}^{\Lambda}|\theta|\right)+\widetilde{B}_{\Lambda} u|\theta| \widetilde{a}_{2}^{\Lambda}+\widetilde{B}_{\Lambda} v \widetilde{a}_{1}^{\Lambda} \frac{\pi}{2} \\
& >-\frac{\pi}{\beta}\left(\widetilde{a}_{1}^{\Lambda}+\widetilde{a}_{2}^{\Lambda}\right)+\widetilde{B}_{\Lambda} u \widetilde{a}_{2}^{\Lambda} \frac{\pi}{6}+\widetilde{B}_{\Lambda} v \widetilde{a}_{1}^{\Lambda} \frac{\pi}{2} \equiv M(u, v) .
\end{aligned}
$$

Furthermore, it turns out that

$$
M(u, v)-r>0
$$

in the domain $S_{3}$. To show this, we observe that the straight line

$$
-\frac{\pi}{2}\left(\widetilde{a}_{1}^{\Lambda}+\widetilde{a}_{2}^{\Lambda}\right)+\widetilde{B}_{\Lambda} u \frac{\pi}{6}+\widetilde{B}_{\Lambda} v \frac{\pi}{2}=\mathrm{const}
$$

passing through the point $u=v=\frac{3}{4 \beta \widetilde{B}}$ remains above the lower branch of the hyperbola $\mathcal{H}_{+}$, and the expression $M(u, v)-r$ takes positive values on this straight line for sufficiently small $\beta$. As the point $(u, v)$ moves "down" from this straight line, the expression $M(u, v)$ also increases. Thus, we must prove that

$$
\frac{1}{\sqrt{r}}\left(\left|L_{1}(u, v)\right|+M(u, v)-r\right)>O(u, v)
$$

in the domain $S_{3}$. To do this we consider the following two cases.

1. $L_{1}(u, v)>0$, that is, we are in the domain lying above the lower branch of the hyperbola $\mathcal{H}_{0}$. In this case, inequality (4.75) is rewritten in the form

$$
L_{1}(u, v)-\sqrt{r} O(u, v)+M(u, v)-r>0 .
$$

This inequality holds in the domain $\widehat{G}_{1}$ lying above the lower branch of the hyperbola

$$
\widehat{\mathcal{H}}_{1}: L(u, v)-\sqrt{r} O(u, v)+M(u, v)-r=0 .
$$

The horizontal asymptote of this hyperbola is

$$
V=\frac{1}{\widetilde{B}_{\Lambda} \beta}-\frac{\pi}{6}+O(\beta)
$$

and lies below the hyperbola $\mathcal{H}_{0}$.

Thus, $\widehat{G}_{1}$ covers the gap between the hyperbolas $\mathcal{H}_{+}$and $\mathcal{H}_{0}$ and, consequently, (4.75) holds in this gap.

2. $L_{1}(u, v)<0$, that is, we are in the domain lying below $\mathcal{H}_{0}$. Rewriting (4.75) in the form

$$
-L(u, v)-\sqrt{r} O(u, v)+M(u, v)-r>0,
$$

it holds in the domain $\widehat{G}_{2}$ bounded from above by the lower branch of the hyperbola

$$
\widehat{H}_{2}:-L(u, v)-\sqrt{r} O(u, v)=0 .
$$

This hyperbola has horizontal asymptote

$$
v=\frac{1}{\beta B}-\sqrt{2} \frac{\bar{c}}{\bar{B}}+O(\beta|\ln \beta|)
$$

and its lower branch lies above the hyperbola $\mathcal{H}_{0}$. Thus, the domain $\widehat{G}_{2}$ covers the gap between $\mathcal{H}_{0}$ and $\mathcal{H}_{-}$and inequality (4.75) also holds in this domain. Thus, the inequality

$$
\left|\Delta_{\Lambda}^{0}(z)\right|>\left|\Delta_{\Lambda}(z)-\Delta_{\Lambda}^{0}(z)\right|
$$

holds for all points of the curve $\mathcal{J}$. By Rouché's theorem, this means that $\Delta_{\Lambda}$ has a unique (real) zero in the domain $\mathcal{F}_{D}^{\Lambda}$. Lemma 4.5 is proved. 
To prove the estimate (3.15), as above we set

$$
u=\widetilde{a}_{1}^{\Lambda} \ln \frac{\tau_{\Lambda}^{(1)}-z}{\beta}, \quad v=\widetilde{a}_{2}^{\Lambda} \ln \frac{\tau_{\Lambda}^{(2)}-z}{\beta} ;
$$

then $u$ and $v$ are connected by the relation

$$
v=V(u)=a_{1}^{\Lambda} \ln \left[\frac{\tau_{\Lambda}^{(2)}-\tau_{\Lambda}^{(1)}}{\beta}+e^{u / a_{2}^{\Lambda}}\right] .
$$

We denote the graph of the function $V(u)$ by $\mathcal{J}$ as before. By repeating the arguments used in the proof of Lemma 4.5 we can show that $\Delta_{\Lambda}(z)>0$ for those $z$ for which the corresponding point $(u, v)$ on $\mathcal{J}$ lies above the horizontal straight line

$$
v=\frac{1}{\beta B}+1
$$

Thus, the zeros $\varepsilon(\Lambda)$ of the determinant $\Delta_{\Lambda}(z)$ satisfy the condition

$$
\widetilde{a}_{2}^{\Lambda} \ln \frac{\tau_{\Lambda}^{(2)}-\varepsilon(\Lambda)}{\beta}=\widetilde{a}_{2}^{\Lambda} \ln \frac{\tau_{\Lambda}^{(2)}-\tau_{\Lambda}^{(1)}+\tau_{\Lambda}^{(1)}-\varepsilon(\Lambda)}{\beta}<\frac{1}{\beta B}+1 .
$$

This inequality immediately implies that

$$
\tau_{\Lambda}^{(1)}-\varepsilon(\Lambda)<\beta \exp \left\{-\frac{1}{\beta\left|\widetilde{B}_{\Lambda}\right| \widetilde{a}_{2}^{\Lambda}}+\frac{1}{\widetilde{a}_{2}^{\Lambda}}\right\}<\exp \left\{-\frac{1}{\beta\left|\widetilde{B}_{\Lambda}\right| \widetilde{a}_{2}^{\Lambda}}\right\}
$$

(for sufficiently small $\beta$ ). Inequality (4.76) now implies that (3.15) holds. Theorem 3.1 is proved.

\section{Proof of Theorem 3.2}

Let $V_{\delta_{1} \beta^{2}}\left(\pi^{\text {caust }}\right)$ be the $\delta_{1} \beta^{2}$-neighbourhood of a caustic (truncated) interval $\pi^{\text {caust }}$, and $V_{\delta_{1} \beta^{2}}( \pm)$ the two parts of it defined in Subsection 3.3. in one of them, $V_{\delta_{1} \beta^{2}}(+)$ (including the interval $\pi^{\text {caust }}$ itself), the minimum is attained at a basic point $k^{\min }(\Lambda)=$ $k_{\text {basic }}^{\sigma}$, and in the other, $V_{\delta_{1} \beta^{2}}(-)$, at a pair point $\left(k_{1}^{\min }, k_{2}^{\min }\right)$, which splits off from a basic point as $\Lambda$ passes across the caustic interval $\pi^{\text {caust }}$. We consider each part of the neighbourhood $V_{\delta_{1} \beta^{2}}\left(\pi^{\text {caust }}\right)$ separately.

1. The case $\Lambda \in V_{\delta_{1} \beta^{2}}(+)$.

Let $U_{\varepsilon}^{\Lambda}\left(k^{\mathrm{min}}\right)$ be the neighbourhood of the minimum point of $\omega_{\Lambda}(k)$ introduced in subsection 3.3. We denote by $\varphi_{\Lambda}(z)$ the integral

$$
\varphi_{\Lambda}(z)=\int_{U_{\varepsilon}^{\Lambda}\left(k^{\min }\right)} \frac{d k}{\omega_{\Lambda}(k)-z} .
$$

Obviously, the following representation holds (see subsection 4.2):

$$
\int_{\mathbb{T}^{2}} \frac{d k}{\omega_{\Lambda}(k)-z}=\varphi_{\Lambda}(z)+\Psi_{\Lambda}^{(1)}(z),
$$

where

$$
\Psi_{\Lambda}^{(1)}(z)=\int_{\mathbb{T}^{2} \backslash U_{\varepsilon}^{\Lambda}\left(k^{\min }\right)} \frac{d k}{\omega_{\Lambda}(k)-z}
$$

is an analytic function of $z \in \mathcal{F}_{D}^{\Lambda}$ satisfying the estimate

$$
\left|\Psi_{\Lambda}^{(1)}(z)\right|<\frac{\bar{C}}{\beta},
$$

where $\bar{C}$ is a constant independent of $z, \Lambda$, and $\beta$.

Recall that in the neighbourhood $U_{\varepsilon}^{\Lambda}\left(k^{\text {min }}\right)$ we introduced the new system of coordinates $x=\left(x^{(1)}, x^{(2)}\right)$ in which the function $\omega_{\Lambda}(k)$ takes the form (3.16). We can assume 
without loss of generality that $\varepsilon<1$. Furthermore, by condition (3.17), the function $\xi(\Lambda)$ occurring in the representation (3.16) satisfies the estimate $|\xi(\Lambda)|<\kappa_{7} \delta_{1} \beta^{2}$ for $\Lambda \in V_{\delta_{1} \beta^{2}}\left(\pi^{\text {caust }}\right)$, and $\xi(\Lambda)>0$ for $\Lambda \in V_{\delta_{1} \beta^{2}}(+)\left(\pi^{\text {caust }}\right)$. Since $\tau_{\Lambda}^{\text {min }}-z<D \beta^{2}$, the integral (5.1) admits the lower estimate

$$
\begin{aligned}
\varphi_{\Lambda}(z) & =\int_{\left(x^{(1)}\right)^{2}+\xi(\Lambda)\left(x^{(2)}\right)^{2}+\left(x^{(2)}\right)^{4}<\varepsilon} \frac{J_{\Lambda}(x) d x}{\beta\left[\left(x^{(1)}\right)^{2}+\xi(\Lambda)\left(x^{(2)}\right)^{2}+\left(x^{(2)}\right)^{4}\right]+\tau_{\Lambda}^{\text {min }}-z} \\
& >C_{1} \int_{\left(x^{(1)}\right)^{2}+\left(\kappa_{7} \delta_{1} \beta^{2}+1\right)\left(x^{(2)}\right)^{2}<\varepsilon} \frac{d x}{\beta\left[\left(x^{(1)}\right)^{2}+\left(\kappa_{7} \delta_{1} \beta^{2}+1\right)\left(x^{(2)}\right)^{2}\right]+D \beta^{2}} \\
& =\frac{C_{1} \pi}{\beta \sqrt{\kappa_{7} \delta_{1} \beta^{2}+1}} \int_{0}^{\sqrt{\varepsilon}} \frac{2 \rho d \rho}{\rho^{2}+D \beta}>K \frac{|\ln \beta|}{\beta},
\end{aligned}
$$

where $K=K(\varepsilon, \delta)>0$ is some constant and $J_{\Lambda}(z)>C_{1}$ is the Jacobian of the change to the new coordinates. Here we have used the fact that the domains satisfy

$$
\begin{aligned}
&\left\{\left(x^{(1)}, x^{(2)}\right):\left(x^{(1)}\right)^{2}+\xi(\Lambda)\left(x^{(2)}\right)^{2}+\left(x^{(2)}\right)^{4}<\varepsilon\right\} \\
& \supset\left\{\left(x^{(1)}, x^{(2)}\right):\left(x^{(1)}\right)^{2}+\left(\kappa_{7} \delta_{1} \beta^{2}+1\right)\left(x^{(2)}\right)^{2}<\varepsilon\right\} .
\end{aligned}
$$

Thus, it follows from (5.2)-(5.4) that

$$
\int_{\mathbb{T}^{2}} \frac{d k}{\omega_{\Lambda}(k)-z}=\varphi_{\Lambda}(z)\left(1+O\left(\frac{1}{|\ln \beta|}\right)\right) .
$$

We now pass to estimating the leading terms $I_{n}^{\Lambda}(z)$ of the series (4.1), which represents the Fredholm determinant $\Delta_{\Lambda}(z)$. Recall that $I_{n}^{\Lambda}(z)$ is represented by the $n$-multiple integral (4.15). Let $\gamma \subseteq\{1, \ldots, n\},|\gamma| \geq 2$, be some subset of indices, and $\mathcal{H}_{\gamma} \subset\left(\mathbb{T}^{2}\right)^{n}$ the set

$$
\mathcal{H}_{\gamma}=\left\{\left(k_{1}, \ldots, k_{n}\right) \in\left(\mathbb{T}^{2}\right)^{n}: k_{i} \in U_{\varepsilon}^{\Lambda}\left(k^{\mathrm{min}}\right), i \in \gamma ; k_{j} \notin U_{\varepsilon}^{\Lambda}\left(k^{\mathrm{min}}\right), j \notin \gamma\right\} .
$$

Consider the integral

$$
I_{n, \gamma}^{\Lambda}(z)=\int_{\mathcal{H}_{\gamma}} \frac{T_{n}^{\Lambda}\left(k_{1}, \ldots, k_{n} ; z\right)}{\prod\left(\omega_{\Lambda}\left(k_{i}\right)-z\right)} \prod d k_{i} .
$$

Let $k_{i_{0}}$ be the nearest point to $k_{\Lambda}^{\text {min }}=k_{\Lambda}^{\text {basic }}$ among the points $\left\{k_{i}, i \in \gamma\right\}$. It follows from Theorem 4.1 that for $\left(k_{1}, \ldots, k_{n}\right) \in \mathcal{H}_{\gamma}$ we have

$$
\begin{aligned}
\left|T_{n}^{\Lambda}\left(k_{1}, \ldots, k_{n} ; z\right)\right| & <\sum_{i_{0} \in \gamma} A\left(C \beta^{2}\right)^{n-1} n^{n / 2} \prod_{i \in \gamma, i \neq i_{0}}\left(k_{i_{0}}-k_{i}\right)^{2} \\
& <\sum_{i_{0} \in \gamma} A\left(C \beta^{2}\right)^{n-1} n^{n / 2} \prod_{i \in \gamma, i \neq i_{0}}\left(\Delta_{i_{0}}-\Delta_{i}\right)^{2} \\
& <\sum_{i_{0} \in \gamma} A\left(C \beta^{2}\right)^{n-1} n^{n / 2} 2^{|\gamma|-1} \prod_{i \in \gamma, i \neq i_{0}} \Delta_{i}^{2},
\end{aligned}
$$

where $\Delta_{i}=k_{i}-k_{\text {basic }}$. Hence we obtain that

$$
\begin{aligned}
\left|I_{n, \gamma}^{\Lambda}\right|< & A\left(2 C \beta^{2}\right)^{n-1} n^{n / 2+1}\left(\int_{U_{\varepsilon}^{\Lambda}} \frac{\left(k-k_{\text {basic }}\right)^{2} d k}{\omega_{\Lambda}(k)-z}\right)^{|\gamma|-1} \\
& \times\left(\int_{\mathbb{T}^{2} \backslash U_{\varepsilon}^{\Lambda}} \frac{d k}{\omega_{\Lambda}(k)-z}\right)^{n-|\gamma|} \int_{U_{\varepsilon}^{\Lambda}} \frac{d k}{\omega_{\Lambda}(k)-z} .
\end{aligned}
$$


Estimating the first integral, we obtain

$$
\begin{aligned}
\int_{U_{\varepsilon}^{\Lambda}} \frac{\left(k-k_{\text {basic }}\right)^{2} d k}{\omega_{\Lambda}(k)-z} & <F^{2} \int_{\left(x^{(1)}\right)^{2}+\xi(\Lambda)\left(x^{(2)}\right)^{2}+\left(x^{(2)}\right)^{4}<\varepsilon} \frac{J(x)|x|^{2} d x}{\beta\left[\left(x^{(1)}\right)^{2}+\left(x^{(2)}\right)^{4}\right]} \\
& <\frac{F^{2} C_{2}}{\beta} \int_{|x|^{2}<\sqrt{\varepsilon}} \frac{|x|^{2} d x}{\left(x^{(1)}\right)^{2}+\left(x^{(2)}\right)^{4}} \\
& =\frac{F^{2} C_{2}}{\beta} \int_{0}^{2 \pi} d \theta \int_{0}^{\varepsilon^{1 / 4}} \frac{\rho^{3} d \rho}{\rho^{2}\left(\sin ^{2} \theta+\rho^{2} \cos ^{2} \theta\right)} \\
& <\frac{F^{2} C_{2}}{\beta} \int_{0}^{\varepsilon^{1 / 4}} \rho d \rho\left[4 \int_{0<\theta<\pi / 4} \frac{d \theta}{\frac{1}{2} \theta^{2}+\frac{1}{4} \rho^{2}}+4 \int_{\pi / 4<\theta<\pi / 2} d \theta\right] \\
& \leq \frac{F^{2} C_{2}}{\beta} \int_{0}^{\varepsilon^{1 / 4}} \rho d \rho\left[\sqrt{2} 8 \frac{1}{\rho} \frac{\pi}{2}+2 \pi\right]=\frac{L}{\beta},
\end{aligned}
$$

where $L=L(\varepsilon)>0$ is a constant. In these estimates we have again used the fact that the domains satisfy

$\left\{\left(x^{(1)}, x^{(2)}\right):\left(x^{(1)}\right)^{2}+\xi(\Lambda)\left(x^{(2)}\right)^{2}+\left(x^{(2)}\right)^{4}<\varepsilon\right\} \subset\left\{\left(x^{(1)}, x^{(2)}\right):\left(x^{(1)}\right)^{2}+\left(x^{(2)}\right)^{2}<\sqrt{\varepsilon}\right\}$.

In (5.9), $F$ is the maximal contraction for the map $f^{-1}$ in the neighbourhood $U_{\varepsilon}^{\Lambda}$ into the domain $G$ (see (3.18)), and $C_{2}$ is a majorant of the Jacobian $J_{\Lambda}(x)$.

From this and from (5.3), (5.6) we find that for $|\gamma| \geq 2$,

$$
\begin{aligned}
\left|I_{n, \gamma}^{\Lambda}\right| & <A\left(2 C \beta^{2}\right)^{n-1} n^{n / 2+1} \varphi_{\Lambda}(z)\left(\frac{L}{\beta}\right)^{|\gamma|-1}\left(\frac{\bar{C}}{\beta}\right)^{n-|\gamma|} \\
& =\frac{A}{L}(2 \widetilde{C} \beta)^{n-1} n^{n / 2+1} \varphi_{\Lambda}(z) L^{|\gamma|} \bar{C}^{n-|\gamma|} .
\end{aligned}
$$

For $\gamma=\varnothing$, it follows from (5.3) and (5.4) that

$$
\left|I_{n, \varnothing}^{\Lambda}\right|<A\left(C \beta^{2}\right)^{n-1} n^{n / 2}\left(\frac{\bar{C}}{\beta}\right)^{n}<\frac{A}{K}(2 C \beta)^{n-1} n^{1+n / 2}(\bar{C})^{n} \varphi_{\Lambda}(z) .
$$

Finally, for $|\gamma|=1$,

$$
\left|I_{n, \gamma}^{\Lambda}\right|<A\left(2 C \beta^{2}\right)^{n-1} n^{1+n / 2} \bar{C}^{n-1} \varphi_{\Lambda}(z) .
$$

From (5.10) - (5.11) we obtain that

$$
\left|I_{n}^{\Lambda}(z)\right|<\bar{A}(2 C \beta)^{n-1} n^{n / 2+1} \sum_{k=0}^{n}\left(\begin{array}{l}
k \\
n
\end{array}\right) \bar{C}^{n-k} \bar{L}^{k}=\bar{A}(2 C \beta)^{n-1} n^{n / 2+1}(L+\bar{C})^{n},
$$

where $\bar{A}=\max \{A, A / K, A / L\}$. Finally this implies that

$$
\sum_{n=2}^{\infty}\left|I_{n}^{\Lambda}\right| \frac{1}{n !}<\operatorname{const} \beta \varphi_{\Lambda}(z) .
$$

It follows from (5.13) and (5.5) that the determinant $\Delta_{\Lambda}(Z)$ for real $z \in \mathcal{F}_{D}^{\Lambda}, \Lambda \in V_{\delta_{1} \beta^{2}}^{(+)}(\pi)$ has the form

$$
\Delta_{\Lambda}(z)=\varphi_{\Lambda}(z)\left(1+O\left(\frac{1}{|\ln \beta|}\right)\right)
$$

and, consequently, does not vanish for sufficiently small $\beta$. 
2. The case $\Lambda \in V_{\delta_{1} \beta^{2}}(-)$. From the representation (3.16) of the function $\omega_{\Lambda}(k)$ in the new coordinates $x=\left(x^{(1)}, x^{(2)}\right)$ in the neighbourhood $U_{\varepsilon}^{\Lambda}$ of the point $k_{\text {basic }}$ it is easy to see that the minimum is attained at two pair points

$$
x_{\text {min }}=\left(0,\left(-\frac{\xi(\Lambda)}{2}\right)^{1 / 2}>0\right) \quad \text { and } \quad \bar{x}_{\min }=\left(0,-\left(-\frac{\xi(\Lambda)}{2}\right)^{1 / 2}<0\right)
$$

and is equal to

$$
\tau_{\Lambda}^{\min }=\omega_{\Lambda}\left(k_{\Lambda}^{\text {basic }}\right)-\beta \frac{\xi^{2}(\Lambda)}{4} .
$$

By condition (3.17) we have $|\xi(\Lambda)|<\kappa_{7} \delta_{1} \beta^{2}$, and the points $x_{\min }$ and $\bar{x}_{\min }$ are inside the neighbourhood

$$
G=\left\{\left(x^{(1)}, x^{(2)}\right):\left(x^{(1)}\right)^{2}+\xi(\Lambda)\left(x^{(2)}\right)^{2}+\left(x^{(2)}\right)^{4}<\varepsilon\right\}
$$

on the plane $\left(x^{(1)}, x^{(2)}\right)$ into which the neighbourhood $U_{\varepsilon}^{\sigma}$ is transformed under the change of coordinates $k \rightarrow x=\left(x^{(1)}, x^{(2)}\right)$. The Taylor expansion of $\omega_{\Lambda}(k)$ in a neighbourhood of the point $x_{\min }$ in powers of the variable

$$
u=x^{(2)}-\left(-\frac{\xi(\Lambda)}{2}\right)^{1 / 2}
$$

has the form

$$
\omega_{\Lambda}(k)=\tau_{\Lambda}^{\min }+\beta\left(\left(x^{(1)}\right)^{2}-u^{2} \xi(\Lambda)+4 u^{3}\left(-\frac{\xi(\Lambda)}{2}\right)^{1 / 2}+u^{4}+\frac{\xi^{(\Lambda)}}{4}\right) .
$$

Hence,

$$
\begin{aligned}
\int_{U_{\varepsilon}^{\Lambda}} & \frac{d k}{\omega_{\Lambda}(k)-z}=\int_{G} \frac{J(x) d x}{\beta\left[\left(x^{(1)}\right)^{2}-u^{2} \xi(\Lambda)+4 u^{3}\left(-\frac{\xi(\Lambda)}{2}\right)^{1 / 2}+u^{4}+\frac{\xi(\Lambda)}{4}\right]+\tau_{\Lambda}^{\min }-z} \\
> & \frac{C_{1}}{\beta} \int_{\left(x^{(1)}\right)^{2}+2 u^{2} \leq \varepsilon / 2} \frac{d x^{(1)} d u}{\left(x^{(1)}\right)^{2}+u^{2}\left(u^{2}-2 \xi(\Lambda)+4 u\left(-\frac{\xi(\Lambda)}{2}\right)^{1 / 2}\right)+\frac{\xi(\Lambda)}{4}+D \beta} \\
& >\frac{C_{1}}{\beta \sqrt{2}} \int_{\left(x^{(1)}\right)^{2}+2 u^{2} \leq \varepsilon / 2, v>0} \frac{d x^{(1)} d v}{\left(x^{(1)}\right)^{2}+2 u^{2}+D \beta}>\bar{K} \frac{\ln \beta}{\beta} .
\end{aligned}
$$

Here we have used the fact that, because $\xi(\Lambda)$ is small and we have assumed that $\varepsilon<1$, the domains satisfy

$$
\left\{\left(x^{(1)}, x^{(2)}\right):\left(x^{(1)}\right)^{2}+2 u^{2}+\frac{\xi(\Lambda)}{4}<\frac{\varepsilon}{2}\right\} \subset G
$$

and

$$
\left(x^{(1)}\right)^{2}-2 u^{2} \xi(\Lambda)+4 u^{3}\left(-\frac{\xi(\Lambda)}{2}\right)^{1 / 2}+u^{4}+\frac{\xi(\Lambda)}{4}<\left(x^{(1)}\right)^{2}+2 u^{2}+\frac{\varepsilon}{2} .
$$

Hence, as above we obtain that

$$
\int_{\mathbb{T}^{2}} \frac{d k}{\omega_{\Lambda}(k)-z}=\varphi_{\Lambda}(z)\left(1+O\left(\frac{1}{|\ln \beta|}\right)\right) .
$$

Now we move on to estimating the leading terms $I_{n}^{\Lambda}$ of the series (4.1). To this end we observe that, since the functions $\omega_{\Lambda}(k)$ and $T_{n}^{\Lambda}\left(k_{1}, \ldots, k_{n} ; z\right)$ are invariant under the involution $j_{\Lambda}: k \rightarrow \Lambda-k$ applied to each argument, the integral $I_{n}^{\Lambda}(z)$ can be represented 
as an $n$-multiple integral over the set $\left(\widetilde{\mathbb{T}}^{2}\right)^{n}$, where $\widetilde{\mathbb{T}}^{2}=\mathbb{T}^{2} / j_{\Lambda}$ is the quotient space of $\mathbb{T}^{2}$ with respect to the involution $j_{\Lambda}$ :

$$
I_{n}^{\Lambda}(z)=2^{n} \int_{\left(\widetilde{\mathbb{T}}^{2}\right)^{n}} \frac{T_{n}^{\Lambda}\left(k_{1}, \ldots, k_{n} ; z\right)}{\prod\left(\omega_{\Lambda}\left(k_{i}\right)-z\right)} \prod d k_{i} .
$$

The neighbourhood $U_{\varepsilon}^{\Lambda}$ is also invariant under $j_{\Lambda}$ and its quotient neighbourhood $\widetilde{U}_{\varepsilon}^{\Lambda}=$ $U_{\varepsilon}^{\Lambda} / j_{\Lambda} \subset \widetilde{\mathbb{T}}^{2}$ can be identified in the new coordinates $\left(x^{(1)}, x^{(2)}\right)$ with the part $\widetilde{G}$ of the domain $G$ given by

$$
\widetilde{G}=\left\{\left(x^{(1)}, x^{(2)}\right) \in G, x^{(2)}>0\right\} .
$$

As above, for each $\gamma=\{1, \ldots, n\},|\gamma| \geq 2$, we define a set $\mathcal{H}_{\gamma}^{n} \subset\left(\widetilde{\mathbb{T}}^{2}\right)^{n}$ as

$$
\mathcal{H}_{\gamma}^{n}=\left\{\left(k_{1}, \ldots, k_{n}\right): k_{i} \in \widetilde{U}_{\varepsilon}^{\Lambda}, i \in \gamma ; k_{j} \notin \widetilde{U}_{\varepsilon}^{\Lambda}, j \in\{1, \ldots, n\} \backslash \gamma\right\} ;
$$

so again we obtain

$$
\left|T_{n}^{\Lambda}\left(k_{1}, \ldots, k_{n} ; z\right)\right|<A\left(C \beta^{2}\right)^{n} n^{n / 2} 2^{|\gamma|-1} \prod_{i \in \gamma, i \neq i_{0}}\left|\Delta_{i}\right|^{2}
$$

for $\left(k_{1}, \ldots, k_{n}\right) \in \mathcal{H}_{\gamma}^{n}$, where $\Delta_{i}=k_{i}-k_{\min }(\Lambda)$ and $i_{0} \in \gamma$ is determined by the condition

$$
\left|\Delta_{i_{0}}\right|=\min _{i \in \gamma}\left\{\left|\Delta_{i}\right|\right\} \text {. }
$$

(Here $\Delta_{i}$ is determined up to a sign, since

$$
k-k_{1}^{\min }=-\left(j_{\Lambda} k-k_{2}^{\min }\right)
$$

for $k \in \mathbb{T}^{2}$, where $k_{1}^{\min }, k_{2}^{\min }$ are pair critical points of $\omega_{\Lambda}(k)$ and $k_{2}^{\min }=j_{\Lambda}\left(k_{1}^{\min }\right)$.)

For the integral $I_{n, \gamma}^{\Lambda}$ defined similarly to (5.7), we derive an estimate analogous to (5.8):

$$
\begin{aligned}
\left|I_{n, \gamma}^{\Lambda}\right|< & A\left(2 C \beta^{2}\right)^{n-1} n^{n / 2+1}\left(\int_{\widetilde{U}_{\varepsilon}^{\Lambda}} \frac{\left(k-k_{\min }(\Lambda)\right)^{2} d k}{\omega_{\Lambda}(k)-z}\right)^{|\gamma|-1} \\
& \times\left(\int_{\widetilde{\mathbb{T}}^{2} \backslash \widetilde{U}_{\varepsilon}^{\Lambda}} \frac{d k}{\omega_{\Lambda}(k)-z}\right)^{n-|\gamma|} \int_{\widetilde{U}_{\varepsilon}^{\Lambda}} \frac{d k}{\omega_{\Lambda}(k)-z} .
\end{aligned}
$$

As above, it remains to estimate the first integral:

$$
\begin{aligned}
& \int_{\widetilde{U}_{\tilde{\varepsilon}}^{\Lambda}} \frac{\left(k-k_{\min }(\Lambda)\right)^{2} d k}{\omega_{\Lambda}(k)-z} \\
& \quad \leq F^{2} C_{2} \int_{\widetilde{G}} \frac{\left(\left(x^{(1)}\right)^{2}+u^{2}\right) d x^{(1)} d u}{\beta\left[\left(x^{(1)}\right)^{2}-2 u^{2} \xi(\Lambda)+4 u^{3}\left(-\frac{\xi(\Lambda)}{2}\right)^{1 / 2}+u^{4}+\frac{\xi(\Lambda)}{4}\right]+\tau_{\Lambda}^{\min }-z},
\end{aligned}
$$

where the neighbourhood $\widetilde{G}$ in the coordinates $\left(x^{(1)}, u=x^{(2)}-\left(-\frac{\xi(\Lambda)}{2}\right)^{1 / 2}\right)$ is written in the form

$$
\begin{aligned}
\widetilde{G}=\left\{\left(x^{(1)}, x^{(2)}\right):\left(x^{(1)}\right)^{2}-2 u^{2} \xi(\Lambda)+4 u^{3}\left(-\frac{\xi(\Lambda)}{2}\right)^{1 / 2}+u^{4}+\frac{\xi(\Lambda)}{4}<\varepsilon,\right. \\
\left.u>-\left(-\frac{\xi(\Lambda)}{2}\right)^{1 / 2}\right\} .
\end{aligned}
$$

Since

$$
-2 u^{2} \xi(\Lambda)+4 u^{3}\left(-\frac{\xi(\Lambda)}{2}\right)^{1 / 2}>0
$$


in the neighbourhood $\widetilde{G}$, this neighbourhood is contained in the neighbourhood

$$
\widetilde{\widetilde{G}}=\left\{\left(x^{(1)}, u\right):\left(x^{(1)}\right)^{2}+u^{4}<\varepsilon\right\}
$$

and thus the right-hand side of (5.15) does not exceed

$$
\frac{F^{2} C_{1}}{\beta} \int_{\widetilde{\widetilde{G}}} \frac{\left(\left(x^{(1)}\right)^{2}+u^{2}\right) d u d x^{(1)}}{\left(\left(x^{(1)}\right)^{2}+u^{4}\right)}<\frac{L}{\beta},
$$

as shown in (5.9). Hence, by repeating the arguments of the preceding subsection, we find that

$$
\sum\left|I_{n}^{\Lambda}(z)\right| \frac{1}{n !}<\operatorname{const} \beta \varphi_{\Lambda}(z) .
$$

As we saw above, this estimate implies that

$$
\Delta_{\Lambda}(z) \neq 0 \quad \text { for } \quad \Lambda \in V_{\delta_{1} \beta^{2}}(-) \quad \text { and } \quad z \in\left(\tau_{\Lambda}^{\min }-D \beta^{2}, \tau_{\Lambda}^{\min }\right) .
$$

Theorem 3.2 is proved.

\section{REFERENCES}

[1] E. L. Lakshtanov and R. A. Minlos, The spectrum of two-particle bound states of transfer matrices of Gibbs fields (an isolated bound state), Funktsional. Anal. i Prilozhen. 38 (2004), no. 3, 52-69; English transl. in Funct. Anal. Appl. 38 (2004), no. 3, 202-216. MR2095134 (2005g:82079)

[2] E. L. Lakshtanov and R. A. Minlos, The spectrum of two-particle bound states of transfer matrices of Gibbs fields (fields on a two-dimensional lattice: adjacent levels), Funktsional. Anal. i Prilozhen. 39 (2005), no. 1, 39-55; English transl. in Funct. Anal. Appl. 39 (2005), no. 1, 31-45. MR2132438 (2006c:82037)

[3] E. A. Kudryavtceva and E. L. Lakshtanov, Classification of singularities and bifurcation of critical points of even functions, Topological methods in Hamiltonian systems, Cambridge Sci. Publ., 2005, $151-174$.

[4] V.I. Arnol'd, S. M. Guseĭn-Zade, and A. N. Varchenko, Singularities of differentiable maps. Vol. II. Monodromy and asymptotics of integrals, Nauka, Moscow, 1984; English transl., Birkhäuser, Boston, MA, 1988. MR0966191 (89g:58024)

[5] E. L. Lakshtanov Leading branches of the transfer matrix spectrum of a general spin model with nearest-neighbor interaction. The high-temperature regime, Vestn. Mosk. Univ., Ser. I 2004 (2004), no. 6, 3-7; English transl. in Mosc. Univ. Math. Bull. 59 (2005), no. 6, 1-5. MR2157594 (2006c:82016)

[6] D. R. Yafaev, Mathematical scattering theory. General theory, St.-Petersburg Univ., 1994; English transl., Transl. of Math. Monographs, vol. 105, Amer. Math. Soc., Providence, RI, 1992. MR1180965 (94f:47012)

[7] Sh. S. Mamatov and R. A. Minlos, Bound states of a two-particle cluster operator, Teoret. Mat. Fiz. 79 (1989), no. 2, 163-179; English transl. in Theoret. and Math. Phys. 79 (1989), no. 2, 455-466. MR.1007792 (90h:82006)

[8] J. Milnor, Morse theory, Princeton Univ. Press, Princeton, N. J., 1963. MR0163331 (29:634)

[9] C. Boldrighini, R. A. Minlos, and A. Pellegrinotti, Random walks in quenched i.i.d. space-time random environment are always a.s. diffusive, Probab. Theory Related Fields 129 (2004), no. 1, 133-156. MR2052866 (2005e:60048)

[10] A. I. Markushevich, A brief course on the theory of analytic functions, Gostekhizdat, Moscow, 1957; English transl., The theory of analytic functions: a brief course, Mir, Moscow, 1983. MR0708893 (84k:30003)

Aveiro University, Portugal

E-mail address: lakshtanov@rambler.ru

Institute for Information Transmission Problems, Russian Academy of Sciences, Moscow, RUssia

E-mail address: minl@iitp.ru

Translated by E. KHUKHRO 